\title{
The Inverse Scattering Transform for the Defocusing Nonlinear Schrödinger Equations with Nonzero Boundary Conditions
}

\author{
By F. Demontis, B. Prinari, C. van der Mee, and F. Vitale
}

[Article amended on April, 2013, after first online publication]

\begin{abstract}
A rigorous theory of the inverse scattering transform for the defocusing nonlinear Schrödinger equation with nonvanishing boundary values $q_{ \pm} \equiv q_{0} e^{i \theta_{ \pm}}$ as $x \rightarrow \pm \infty$ is presented. The direct problem is shown to be well posed for potentials $q$ such that $q-q_{ \pm} \in L^{1,2}\left(\mathbb{R}^{ \pm}\right)$, for which analyticity properties of eigenfunctions and scattering data are established. The inverse scattering problem is formulated and solved both via Marchenko integral equations, and as a Riemann-Hilbert problem in terms of a suitable uniform variable. The asymptotic behavior of the scattering data is determined and shown to ensure the linear system solving the inverse problem is well defined. Finally, the triplet method is developed as a tool to obtain explicit multisoliton solutions by solving the Marchenko integral equation via separation of variables.
\end{abstract}

\section{Introduction}

Nonlinear Schrödinger (NLS) systems have attracted the attention of the physical and mathematical communities since the 1950s, with the early work of Ginzburg, Landau, and Pitaevskii on the macroscopic theories of superconductivity and superfluidity [1-3]. Nonetheless, it was not until the works of Chiao et al. [4] and Talanov [5,6] that the wider physical importance of NLS equations became evident, especially in connection with the

Address for correspondence: Barbara Prinari, Department of Mathematics, University of Colorado at Colorado Springs, CO, USA; Dipartimento di Matematica e Fisica "E. De Giorgi," Università del Salento, Lecce, Italy; e-mail: bprinari@uccs.edu 
phenomena of self-focusing/self-defocusing and the conditions under which an electromagnetic beam can propagate in nonlinear media without spreading. Equations of NLS-type have since then been derived in such diverse fields as deep water waves (cf. [7, 8]), plasma physics [9], nonlinear fiber optics $[10,11]$, magnetic spin waves [12, 13], Bose-Einstein condensates [14], etc. As a matter of fact, most dispersive energy preserving systems give rise, in appropriate limits, to the scalar NLS, which explains the keen interest in NLS as a prototypical integrable system and motivates the effort put into advancing our mathematical understanding of this equation.

The inverse scattering transform (IST) as a method to solve the initial-value problem for the scalar NLS equation

$$
i q_{t}=q_{x x}-2 \sigma|q|^{2} q
$$

(subscripts $x$ and $t$ denote partial differentiation throughout) has been extensively studied in the literature, both in the focusing $(\sigma=-1)$ and in the defocusing $(\sigma=1)$ dispersion regimes (see, for instance, [7, 15-18] for detailed accounts of the IST in the case of potentials $q(x, t)$ rapidly decaying as $|x| \rightarrow \infty)$. The situation is quite different when one is interested in potentials that do not decay as $|x| \rightarrow \infty$. This class of potentials is particularly important for the defocusing NLS, since it admits soliton solutions with nonzero boundary conditions (NZBCs), the so-called dark/gray solitons, which have the form:

$$
q(x, t)=q_{0} e^{2 i q_{0}^{2} t}\left[\cos \alpha+i(\sin \alpha) \tanh \left[q_{0}(\sin \alpha)\left(x-2 q_{0} t \cos \alpha-x_{0}\right)\right]\right]
$$

with $q_{0}, \alpha$, and $x_{0}$ arbitrary real parameters. Dark soliton solutions are such that $|q(x, t)| \rightarrow q_{0}$ as $x \rightarrow \pm \infty$, and appear as localized dips of intensity $q_{0}^{2} \sin ^{2} \alpha$ on the background field $q_{0}$. The IST for the defocusing NLS equation with NZBCs was first studied in 1973 [19]; the problem was subsequently clarified and generalized in various works [20-27], and a detailed study can be found in the monograph [28].

Even though the IST for the defocusing NLS with NZBCs was first presented almost 40 years ago, many important issues still remain to be clarified, i.e.,: (i) No attempt has been made so far to identify the most suitable functional class of nondecaying potentials where the direct and inverse scattering problems can be solved, or to rigorously establish the analyticity properties of eigenfunctions and scattering data. In that respect, (the analog of) Schwartz class is usually assumed for the potential (cf., for instance, [23, 28, 29]), which is clearly unnecessarily restrictive. In $[24,25]$ the issue of establishing the analyticity of the eigenfunctions was addressed by reformulating the scattering problem in terms of a so-called energy dependent potential, but the drawback of that approach is a very complicated dependence of eigenfunctions and data on the scattering parameter. (ii) For the focusing NLS in the vanishing case, it has been shown in $[30,31]$ that there are no discrete eigenvalues and no spectral singularities if $\|q\|_{1}<\pi / 2$, where the $\pi / 2$ bound is optimal. Whether the 
existence of discrete eigenvalues for the defocusing NLS in the nonvanishing case can be related to the equivalent of an area theorem for the initial profile of the solution as a dip of intensity on the background field is still an open issue. (iii) As far as the inverse problem is concerned, it was first formulated (but not solved) as a Riemann-Hilbert problem in [28], without any investigation on its well-posedness.

In this work we will address many of the above mentioned open issues, and also some related important topics. The problem of the existence of an area theorem will be a subject for future investigation. The plan of the paper is outlined below. We study the IST for the scalar defocusing NLS [Equation (1) with $\sigma=1]$ :

$$
i q_{t}=q_{x x}-2|q|^{2} q
$$

with NZBCs

$$
q(x, t) \rightarrow q_{ \pm}(t)=q_{0} e^{2 i q_{0}^{2} t+i \theta_{ \pm}} \quad \text { as } \quad x \rightarrow \pm \infty,
$$

where $q_{0}>0$ and $0 \leq \theta_{ \pm}<2 \pi$ are arbitrary constants. Sections 2 and 3 are devoted to the study of the direct scattering problem. We will prove that it is well defined for potentials $q$ such that $q-q_{ \pm} \in L^{1,2}\left(\mathbb{R}^{ \pm}\right), L^{1, s}(\mathbb{R})$ being the complex Banach space of all measurable functions $f(x)$ for which $(1+|x|)^{s} f(x)$ is integrable. We will obtain integral representations for the scattering data, and establish analyticity of eigenfunctions and scattering data for potentials in this class. Moreover, we will prove that, under the assumption that $q-q_{ \pm} \in L^{1,4}\left(\mathbb{R}^{ \pm}\right)$, the discrete eigenvalues are finite in number and belong to the spectral gap $k \in\left(-q_{0}, q_{0}\right)$ (see Appendix B for details). In Section 4 we will formulate and solve the inverse problem as a Riemann-Hilbert problem (RHP) in terms of a suitable uniform variable, and we will show that the asymptotic behavior of the scattering data ensures the algebraic-integral system of equations providing the solution of the inverse problem is well defined. Finally, in Section 5 we will formulate the inverse problem in terms of Marchenko integral equations, and we will develop the triplet method as a tool to obtain explicit multisoliton solutions by solving the Marchenko equations via separation of variables [32-35]. The crux of the method is to represent the Marchenko kernel as $C e^{-(y+z) A} B$, where $(A, B, C)$ is a suitable matrix triplet. The NLS solutions obtained in this way will not contain anything more complicated than matrix exponentials and solutions of Lyapunov equations, and, if necessary, can hence be "unzipped" into (lengthy) expressions containing elementary functions, or used as input to numerical calculations. More technical proofs will be deferred to the Appendices, while for results that are already well established in the literature, such as, for instance, the time evolution of eigenfunctions and scattering data, we will simply refer the reader to the appropriate references.

Some of the results presented in this paper will be relevant in the context of recent theoretical studies and experimental observations of defocusing NLS in 
the framework of dispersive shock waves in optical fibers [see, for instance, [36] regarding the appearance and evolution of dispersive shock waves when an input (reflectionless) pulse containing a large number of dark or gray solitons is injected in the fiber]. Importantly, the present work will pave the way for generalizing similar results to the defocusing vector NLS equation, for which the IST with NZBCs was recently developed (cf. [37] for the two-component vector NLS and [38] for vector NLS with an arbitrary number of components).

\section{Direct problem: $k, \lambda$ variables}

It is well known that Equation (3) can be associated to the so-called ZS-AKNS scattering problem [8], [15]:

$$
\frac{\partial X}{\partial x}(x, k)=\left(-i k \sigma_{3}+Q(x)\right) X(x, k), \quad x \in \mathbb{R},
$$

where

$$
\sigma_{3}=\left(\begin{array}{cc}
1 & 0 \\
0 & -1
\end{array}\right), \quad Q(x)=\left(\begin{array}{cc}
0 & q(x) \\
q^{*}(x) & 0
\end{array}\right),
$$

$q(x)$ is the potential, and $k$ is a complex spectral parameter. We start off by assuming that $q(x)-q_{ \pm}$belongs to $L^{1}\left(\mathbb{R}^{ \pm}\right)$, although in the course of this article we will somewhat strengthen the integrability requirements.

The direct problem for the system (5) with boundary conditions (4) is more complicated than the counterpart with vanishing boundary conditions [i.e., $q(x, t) \rightarrow 0$ as $x \rightarrow \pm \infty$ ]. In fact, when looking for asymptotic eigenvalues and eigenvectors of the scattering problem, one has to deal with the new spectral variable $\lambda=\sqrt{k^{2}-q_{0}^{2}}$ (see, for instance, [19, 28, 37]). The variable $k$ is then thought of as belonging to a Riemann surface $\mathbb{K}$ consisting of a sheet $\mathbb{K}^{+}$and a sheet $\mathbb{K}^{-}$which both coincide with the complex plane cut along the semilines

$$
\Sigma=\left(-\infty,-q_{0}\right] \cup\left[q_{0}, \infty\right)
$$

with its edges glued in such a way that $\lambda(k)$ is continuous through the cut. The variable $\lambda$ is thought of as belonging to the complex plane consisting of the upper half complex plane $\Lambda^{+}$and the lower half complex plane $\Lambda^{-}$ glued together along the full real line. The transformation $k \mapsto \lambda$ maps $\mathbb{K}^{ \pm}$ onto $\Lambda^{ \pm}$, the cut $\Sigma$ onto the real line, and the points $\pm q_{0}$ to zero. Moreover, $\{\lambda+k, \lambda-k\} \subset \Lambda^{ \pm}$for any $k \in \mathbb{K}^{ \pm}$.

For later convenience, we write (5) in the form

$$
\frac{\partial X}{\partial x}(x, k)=A_{ \pm}(k) X(x, k)+\left(Q(x)-Q_{ \pm}\right) X(x, k),
$$


where

$$
A_{ \pm}(k)=-i k \sigma_{3}+Q_{ \pm} \equiv\left(\begin{array}{cc}
-i k & q_{ \pm} \\
q_{ \pm}^{*} & i k
\end{array}\right), \quad Q_{ \pm}=\left(\begin{array}{cc}
0 & q_{ \pm} \\
q_{ \pm}^{*} & 0
\end{array}\right)
$$

such that

$$
\begin{aligned}
& A_{ \pm}(k)=-\sigma_{3} A_{ \pm}^{\dagger}\left(k^{*}\right) \sigma_{3}, \\
& A_{ \pm}^{*}\left(k^{*}\right)=\sigma_{1} A_{ \pm}(k) \sigma_{1} .
\end{aligned}
$$

Here the dagger and asterisk denote, respectively, the complex conjugate transpose and the complex conjugate, and $\sigma_{1}$ is the first Pauli matrix

$$
\sigma_{1}=\left(\begin{array}{ll}
0 & 1 \\
1 & 0
\end{array}\right)
$$

We remark that (8) is equivalent to the following scattering problem:

$$
\frac{\partial X}{\partial x}(x, k)=A(x, k) X(x, k)+\left(Q(x)-Q_{f}(x)\right) X(x, k),
$$

where we have defined

$$
A(x, k)=\theta(x) A_{+}(k)+\theta(-x) A_{-}(k), \quad Q_{f}(x)=\theta(x) Q_{+}+\theta(-x) Q_{-} .
$$

$Q_{f}(x)$ is the analog of the free potential, and $\theta(x)$ denotes the Heaviside function $[\theta(x)=1$ for $x \geq 0$ and zero otherwise]. We also point out that the scattering problem (10) is continuous at $x=0$ if $X(x, k)$ and $Q(x)$ are, and it coincides with (5). Since we only study solutions to (10) satisfying certain asymptotic conditions, these solutions are defined in the weak sense as solutions of "equivalent" integral equations.

Clearly, the ZS-AKNS Hamiltonian operator $H=i \sigma_{3}(d / d x-Q)$ is selfadjoint on the orthogonal direct sum of two copies of $L^{2}(\mathbb{R})$. It is convenient to introduce the free Hamiltonian

$$
H_{f}=i \sigma_{3}\left(\frac{d}{d x}-Q_{f}\right),
$$

and to formulate the scattering theory as a result of the potential difference $Q-Q_{f}$ occurring in the full Hamiltonian $H$.

When studying Equation (5) with vanishing boundary conditions, it is customary to define Jost solutions as particular, asymptotically free column vector solutions and to group them together as columns of fundamental eigensolutions. The generalization of these definitions to Equation (5) with NZBCs (4) is more involved. In the next two subsections, we will define fundamental solutions and Jost solutions, point out how they are related, and determine their analyticity properties. Alongside, we will define the transition coefficient matrices coupling the fundamental eigensolutions as $x \rightarrow \pm \infty$, as well as the scattering coefficients. 


\subsection{Fundamental eigenfunctions}

Taking $k \in \Sigma$ as in (7), we define the fundamental eigenfunctions $\tilde{\Psi}(x, k)$ from the right and $\tilde{\Phi}(x, k)$ from the left as those square matrix solutions to (5) [or (10)] satisfying

$$
\begin{aligned}
& \tilde{\Psi}(x, k)=e^{x A_{+}(k)}\left[I_{2}+o(1)\right], \quad x \rightarrow+\infty, \\
& \tilde{\Phi}(x, k)=e^{x A_{-}(k)}\left[I_{2}+o(1)\right], \quad x \rightarrow-\infty .
\end{aligned}
$$

Here $I_{p}$ denotes the identity matrix of order $p$.

For later use, we determine the fundamental matrix $\mathcal{G}(x, y ; k)$ for the scattering problem with generator $A(x, k)$ defined in (11). In other words, we look for the weak solution $\mathcal{G}(x, y ; k)$ of the system (5) [or (10)] with potential $Q(x)=Q_{f}(x)$ such that

$$
\begin{aligned}
\frac{\partial}{\partial x} \mathcal{G}(x, y ; k) & =A(x, k) \mathcal{G}(x, y ; k), \\
\mathcal{G}(x, x ; k) & =I_{2} .
\end{aligned}
$$

As shown in Appendix A (see also [39]), one has

$$
\mathcal{G}(x, y ; k)= \begin{cases}e^{(x-y) A_{+}(k)}, & x, y \geq 0 \\ e^{(x-y) A_{-}(k)}, & x, y \leq 0 \\ e^{x A_{+}(k)} e^{-y A_{-}(k)}, & x,-y \geq 0, \\ e^{x A_{-}(k)} e^{-y A_{+}(k)}, & x,-y \leq 0 .\end{cases}
$$

In particular, from (13) it follows

$$
\begin{aligned}
& \mathcal{G}(x, 0 ; k)=\theta(x) e^{x A_{+}(k)}+\theta(-x) e^{x A_{-}(k)}, \\
& \mathcal{G}(0, y ; k)=\theta(y) e^{-y A_{+}(k)}+\theta(-y) e^{-y A_{-}(k)} .
\end{aligned}
$$

As a result, $\mathcal{G}(x, y ; k)$ is a square matrix which depends continuously on $(x, y, k) \in \mathbb{R}^{2} \times \Sigma$ and satisfies (5) with the free potential $Q_{f}$. Recalling (9), for $k \in \Sigma$ we have the symmetry relations

$$
\begin{gathered}
\mathcal{G}^{\dagger}(x, y ; k)=-\sigma_{3} \mathcal{G}(y, x ; k) \sigma_{3}, \\
\mathcal{G}^{*}(x, y ; k)=\sigma_{1} \mathcal{G}(x, y ; k) \sigma_{1} .
\end{gathered}
$$

Moreover, $A_{ \pm}(k)$ have the two distinct imaginary eigenvalues $\pm i \lambda$ if $k \in \Sigma \backslash\left\{ \pm q_{0}\right\}$, and are nondiagonalizable with a double zero eigenvalue if $k= \pm q_{0}$. Further, the matrix groups $e^{x A_{ \pm}(k)}$ are bounded in $x \in \mathbb{R}$ if $k \in \Sigma \backslash\left\{ \pm q_{0}\right\}$, and grow linearly in $x \in \mathbb{R}$ in any matrix norm if $k= \pm q_{0}$. In particular, one has

$$
e^{x A_{ \pm}\left(q_{0}\right)}=\left(\begin{array}{cc}
1-i q_{0} x & q_{ \pm} x \\
q_{ \pm}^{*} x & 1+i q_{0} x
\end{array}\right), \quad e^{x A_{ \pm}\left(-q_{0}\right)}=\left(\begin{array}{cc}
1+i q_{0} x & q_{ \pm} x \\
q_{ \pm}^{*} x & 1-i q_{0} x
\end{array}\right)
$$


and thus there exists a constant $C \geq 1$ (independent of $(x, y) \in \mathbb{R}^{2}$ ) such that

$$
\|\mathcal{G}(x, y ; k)\| \leq \begin{cases}C, & k<-q_{0} \text { or } k>q_{0}, \\ C(1+|x|)(1+|y|), & k= \pm q_{0} .\end{cases}
$$

Proposition 1. Suppose the entries of $Q(x)-Q_{f}(x)$ belong to $L^{1}(\mathbb{R})$ (equivalently, $q(x)-q_{ \pm} \in L^{1}\left(\mathbb{R}^{ \pm}\right)$). Then for $k \in \Sigma \backslash\left\{ \pm q_{0}\right\}$ the Volterra integral equations

$$
\begin{aligned}
& \tilde{\Psi}(x, k)=\mathcal{G}(x, 0 ; k)-\int_{x}^{\infty} d y \mathcal{G}(x, y ; k)\left[Q(y)-Q_{f}(y)\right] \tilde{\Psi}(y, k), \\
& \tilde{\Phi}(x, k)=\mathcal{G}(x, 0 ; k)+\int_{-\infty}^{x} d y \mathcal{G}(x, y ; k)\left[Q(y)-Q_{f}(y)\right] \tilde{\Phi}(y, k),
\end{aligned}
$$

have the fundamental eigenfunctions defined by (12) as their unique solutions. The same conclusion holds for $k= \pm q_{0}$ if the entries of $Q(x)-Q_{f}(x)$ are in $L^{1,2}(\mathbb{R})$, i.e., if $(1+|x|)^{2}\left[q(x)-q_{ \pm}\right]$belong to $L^{1}\left(\mathbb{R}^{ \pm}\right)$.

Proof. According to (17), if $k \in \Sigma \backslash\left\{ \pm q_{0}\right\}$, the iterates of (18a) are bounded above in the matrix norm by the iterates of the integral inequality

$$
\|\tilde{\Psi}(x, k)\| \leq C+C \int_{x}^{\infty} d y\left\|Q(y)-Q_{f}(y)\right\|\|\tilde{\Psi}(y, k)\|,
$$

which, by Gronwall's inequality, implies

$$
\|\tilde{\Psi}(x, k)\| \leq C \exp \left(C \int_{x}^{\infty} d y\left\|Q(y)-Q_{f}(y)\right\|\right) \leq C e^{C\left\|Q-Q_{f}\right\|_{1}}<+\infty .
$$

For $k= \pm q_{0}$, by using the second inequality in (17) we have instead

$$
\frac{\|\tilde{\Psi}(x, k)\|}{C(1+|x|)} \leq 1+C \int_{x}^{\infty} d y(1+|y|)^{2}\left\|Q(y)-Q_{f}(y)\right\| \frac{\|\tilde{\Psi}(y, k)\|}{C(1+|y|)} .
$$

Then, by Gronwall's inequality,

$$
\|\tilde{\Psi}(x, k)\| \leq C(1+|x|) \exp \left(C \int_{x}^{\infty} d y(1+|y|)^{2}\left\|Q(y)-Q_{f}(y)\right\|\right) .
$$

We recall that the unique solution of (18a) can be obtained by considering the iterative (uniformly convergent) scheme:

$$
\tilde{\Psi}(x, k)=\sum_{n=0}^{\infty} \tilde{\Psi}_{n}(x, k)
$$


with

$$
\begin{aligned}
\tilde{\Psi}_{0}(x, k) & =\mathcal{G}(x, 0 ; k), \\
\tilde{\Psi}_{n+1}(x, k) & =-\int_{x}^{\infty} d y \mathcal{G}(x, y ; k)\left[Q(y)-Q_{f}(y)\right] \tilde{\Psi}_{n}(y, k),
\end{aligned}
$$

where

$$
\left\|\tilde{\Psi}_{n}(x, k)\right\| \leq C(1+|x|) \frac{1}{n !}\left(C \int_{x}^{\infty} d y(1+|y|)^{2}\left\|Q(y)-Q_{f}(y)\right\|\right)^{n} .
$$

The statement for $(18 b)$ is proved analogously.

Proposition 1 implies that for $k \in \Sigma$

$$
\begin{gathered}
\tilde{\Psi}(x, k)=\mathcal{G}(x, 0 ; k)\left[\mathbb{A}_{l}(k)+o(1)\right], \quad x \rightarrow-\infty, \\
\tilde{\Phi}(x, k)=\mathcal{G}(x, 0 ; k)\left[\mathbb{A}_{r}(k)+o(1)\right], \quad x \rightarrow+\infty,
\end{gathered}
$$

where the transition coefficient matrices $\mathbb{A}_{l}(k)$ and $\mathbb{A}_{r}(k)$ are given by

$$
\begin{aligned}
& \mathbb{A}_{l}(k)=I_{2}-\int_{-\infty}^{\infty} d y \mathcal{G}(0, y ; k)\left[Q(y)-Q_{f}(y)\right] \tilde{\Psi}(y, k), \\
& \mathbb{A}_{r}(k)=I_{2}+\int_{-\infty}^{\infty} d y \mathcal{G}(0, y ; k)\left[Q(y)-Q_{f}(y)\right] \tilde{\Phi}(y, k),
\end{aligned}
$$

obtained using the multiplication property (A.5). The transition coefficient matrices are continuous for $k \in \Sigma \backslash\left\{ \pm q_{0}\right\}$ if the entries of $Q(x)-Q_{f}(x)$ belong to $L^{1}(\mathbb{R})$, and for $k \in \Sigma$ if the entries of $(1+|x|)^{2}\left[Q(x)-Q_{f}(x)\right]$ belong to $L^{1}(\mathbb{R})$.

Using that $Q^{*}(x)=\sigma_{1} Q(x) \sigma_{1}$ and $\sigma_{3} \sigma_{1}=-\sigma_{1} \sigma_{3}$, we easily derive that $\sigma_{1} X^{*}\left(x, k^{*}\right)$ is a solution to Equation (5) if $X(x, k)$ is. From (9c) and the boundary conditions (12) we thus get the symmetry relations

$$
\sigma_{1} \tilde{\Psi}^{*}\left(x, k^{*}\right) \sigma_{1}=\tilde{\Psi}(x, k), \quad \sigma_{1} \tilde{\Phi}^{*}\left(x, k^{*}\right) \sigma_{1}=\tilde{\Phi}(x, k) .
$$

We can also easily prove the following.

PROPOSITION 2. For $k \in \Sigma$

$$
\begin{aligned}
& \mathbb{A}_{l}(k)=\mathbb{A}_{r}^{-1}(k)=\sigma_{3} \mathbb{A}_{r}^{\dagger}(k) \sigma_{3}, \quad \mathbb{A}_{r}(k)=\mathbb{A}_{l}^{-1}(k)=\sigma_{3} \mathbb{A}_{l}^{\dagger}(k) \sigma_{3}, \\
& \mathbb{A}_{l}(k)=\sigma_{1} \mathbb{A}_{l}^{*}(k) \sigma_{1}, \quad \mathbb{A}_{r}(k)=\sigma_{1} \mathbb{A}_{r}^{*}(k) \sigma_{1} .
\end{aligned}
$$

The above relations also hold for $k \in \mathbb{K}$, with the arguments of $\mathbb{A}_{l, r}^{\dagger}$ and $\mathbb{A}_{l, r}^{*}$ replaced by $k^{*}$, whenever the involved transition coefficients can be continued off $\Sigma$. 
Proof. Let $Z(x, k)$ and $\tilde{Z}(x, k)$ be two square matrix solutions of (5). For $k \in \mathbb{R}$ we obviously have

$$
\frac{\partial \tilde{Z}^{\dagger}}{\partial x}-\tilde{Z}^{\dagger} Q=i k \tilde{Z}^{\dagger} \sigma_{3}
$$

As a result,

$$
\begin{aligned}
\frac{\partial}{\partial x}\left[\tilde{Z}^{\dagger} \sigma_{3} Z\right] & =\tilde{Z}^{\dagger} \sigma_{3} \frac{\partial Z}{\partial x}+\frac{\partial \tilde{Z}^{\dagger}}{\partial x} \sigma_{3} Z \\
& =\tilde{Z}^{\dagger} \sigma_{3}\left(-i k \sigma_{3}+Q\right) Z+\tilde{Z}^{\dagger}\left(i k \sigma_{3}+Q\right) \sigma_{3} Z \\
& =\tilde{Z}^{\dagger}\left(\sigma_{3} Q+Q \sigma_{3}\right) Z=0,
\end{aligned}
$$

which implies that $\tilde{Z}^{\dagger}(x, k) \sigma_{3} Z(x, k)$ is independent of $x \in \mathbb{R}$. Using the asymptotic properties of the two fundamental eigenfunctions as $x \rightarrow \pm \infty$, (22a) follow. Equations (22b) follow directly from (19), (21), and (15b). The result for $k \notin \Sigma$ follows from Schwarz reflection principle.

Let us denote the transition matrices as follows:

$$
\mathbb{A}_{l}(k)=\left(\begin{array}{ll}
A_{l 1}(k) & A_{l 2}(k) \\
A_{l 3}(k) & A_{l 4}(k)
\end{array}\right), \quad \mathbb{A}_{r}(k)=\left(\begin{array}{ll}
A_{r 1}(k) & A_{r 2}(k) \\
A_{r 3}(k) & A_{r 4}(k)
\end{array}\right) .
$$

Then Equations (22a) imply that for $k \in \Sigma$ we have

$$
\begin{aligned}
& \left|A_{l 1}(k)\right| \geq 1, \quad\left|A_{r 1}(k)\right| \geq 1, \quad A_{l 1}(k)=A_{r 1}^{*}(k), \\
& \left|A_{l 4}(k)\right| \geq 1, \quad\left|A_{r 4}(k)\right| \geq 1, \quad A_{l 4}(k)=A_{r 4}^{*}(k) .
\end{aligned}
$$

To prove (25) we proceed as follows. First of all, we observe that from (22a) we can write

$$
\sigma_{3}=\mathbb{A}_{l}(k) \sigma_{3} \mathbb{A}_{l}^{\dagger}(k)
$$

As a consequence, we get

$$
\mathbb{A}_{l}(k) \sigma_{3} \mathbb{A}_{l}^{\dagger}(k)=\left(\begin{array}{ll}
A_{l 1}(k) & A_{l 2}(k) \\
A_{l 3}(k) & A_{l 4}(k)
\end{array}\right)\left(\begin{array}{cc}
A_{l 1}^{*}(k) & A_{l 3}^{*}(k) \\
-A_{l 2}^{*}(k) & -A_{l 4}^{*}(k)
\end{array}\right)=\left(\begin{array}{cc}
1 & 0 \\
0 & -1
\end{array}\right)
$$

and the last equation implies that

$$
\left|A_{l 1}\right|^{2}=1+\left|A_{l 2}\right|^{2} \geq 1, \quad\left|A_{l 4}\right|^{2}=1+\left|A_{l 3}\right|^{2} \geq 1 .
$$

The proof for the entries of $\mathbb{A}_{r}(k)$ can be carried out in a similar way.

Using (23), we easily prove that for each $x \in \mathbb{R}$

$$
\tilde{\Psi}^{\dagger}(x, k) \sigma_{3} \tilde{\Psi}(x, k)=\tilde{\Phi}^{\dagger}(x, k) \sigma_{3} \tilde{\Phi}(x, k)=-\sigma_{3} .
$$

As a result, the diagonal entries of the transition matrices cannot vanish for $k \in \Sigma$.

It is worth mentioning that instead of deriving the fundamental eigenfunctions of (5) from those pertaining to the free Hamiltonian, we can also derive them 
as perturbations of $e^{x A_{ \pm}(k)}$ as $x \rightarrow \pm \infty$. In that case, we obtain the integral equations

$$
\begin{aligned}
& \tilde{\Psi}(x, k)=e^{x A_{+}(k)}-\int_{x}^{\infty} d y e^{(x-y) A_{+}(k)}\left[Q(y)-Q_{+}\right] \tilde{\Psi}(y, k), \\
& \tilde{\Phi}(x, k)=e^{x A_{-}(k)}+\int_{-\infty}^{x} d y e^{(x-y) A_{-}(k)}\left[Q(y)-Q_{-}\right] \tilde{\Phi}(y, k) .
\end{aligned}
$$

Equations (18a) and (26a) coincide if $x \geq 0$, whereas (18b) and (26b) coincide if $x \leq 0$. In all other cases, the corresponding integral Equations (18) and (26) are quite different, though their solutions coincide (in fact, $\tilde{\Psi}(x, k)$ and $\tilde{\Phi}(x, k)$ both satisfy (10) with the asymptotic conditions (12a) and (12b), respectively). Equations (26), however, are not suitable for investigating the behavior of the eigenfunctions as $x \rightarrow \mp \infty$. In fact, the iterates of (26a) (resp., (26b)) are continuous functions of $x \in \mathbb{R}$ which converge uniformly to $\tilde{\Psi}(x, k)$ (resp., $\tilde{\Phi}(x, k)$ ) for $x \geq x_{0}>-\infty$ (resp., $x \leq x_{0}<+\infty$ ), but nothing can be said about the limit as $x \rightarrow-\infty$ (resp. $x \rightarrow+\infty$ ).

\subsection{Jost solutions}

The fundamental eigenfunctions introduced in Section 2.1 are not scattering eigenfunctions, in the sense that their asymptotic behavior as $x \rightarrow \pm \infty$ couples both asymptotic eigenvalues $\pm i \lambda$ of the scattering problem. In this subsection we introduce the Jost solutions as column vector solutions to (10) and establish their analyticity properties. To this aim, we multiply from the right the fundamental eigensolutions $\tilde{\Psi}(x, k)$ and $\tilde{\Phi}(x, k)$ by the eigenvectors of $A_{ \pm}(k)$ corresponding to the eigenvalues $i \lambda$ and $-i \lambda$, i.e., by the columns of

$$
W_{ \pm}(k)=\left(\begin{array}{cc}
\lambda+k & \lambda-k \\
i q_{ \pm}^{*} & -i q_{ \pm}^{*}
\end{array}\right),
$$

with det $W_{ \pm}(k)=-2 i q_{ \pm}^{*} \lambda$ and

$$
A_{ \pm}(k) W_{ \pm}(k)=W_{ \pm}(k) \operatorname{diag}(-i \lambda, i \lambda) .
$$

Moreover, it is easily verified that for $k \in \Sigma \backslash\left\{ \pm q_{0}\right\}$ we have

$$
\left[W_{ \pm}(k) D^{-1 / 2}(k)\right]^{\dagger}=\sigma_{3}\left[W_{ \pm}(k) D^{-1 / 2}(k)\right]^{-1} \sigma_{3},
$$

where $D(k)=\operatorname{diag}(2 \lambda(\lambda+k), 2 \lambda(k-\lambda))$. We now call the columns of

$$
\begin{aligned}
& \tilde{\Psi}(x, k) W_{+}(k)=(\bar{\psi}(x, k) \quad \psi(x, k)), \\
& \tilde{\Phi}(x, k) W_{-}(k)=(\phi(x, k) \quad \bar{\phi}(x, k)),
\end{aligned}
$$


the Jost solutions from the right and the left, respectively. In fact, for the Jost solutions we get

$$
\begin{aligned}
& \bar{\psi}(x, k) \sim e^{-i \lambda x}\left(\begin{array}{c}
\lambda+k \\
i q_{+}^{*}
\end{array}\right), \quad \psi(x, k) \sim e^{i \lambda x}\left(\begin{array}{c}
\lambda-k \\
-i q_{+}^{*}
\end{array}\right), \quad x \rightarrow+\infty, \\
& \phi(x, k) \sim e^{-i \lambda x}\left(\begin{array}{c}
\lambda+k \\
i q_{-}^{*}
\end{array}\right), \quad \bar{\phi}(x, k) \sim e^{i \lambda x}\left(\begin{array}{c}
\lambda-k \\
-i q_{-}^{*}
\end{array}\right), \quad x \rightarrow-\infty .
\end{aligned}
$$

Using that $\sigma_{1} X^{*}\left(x, k^{*}\right)$ is a solution to Equation (5) if $X(x, k)$ is, we easily derive the symmetry relations

$$
\bar{\phi}(x, k)=\frac{-i q_{-}^{*}}{\lambda+k} \sigma_{1} \phi^{*}\left(x, k^{*}\right), \quad \psi(x, k)=\frac{-i q_{+}^{*}}{\lambda+k} \sigma_{1} \bar{\psi}^{*}\left(x, k^{*}\right) .
$$

Moreover, replacing $k \in \mathbb{K}^{ \pm}$by the "same" $k \in \mathbb{K}^{\mp}$ and hence replacing $\lambda \in \Lambda^{ \pm}$by $-\lambda \in \Lambda^{\mp}$, we convert $\bar{\psi}(x, k)$ into $-\psi(x, k)$ and $\phi(x, k)$ into $-\bar{\phi}(x, k)$ (symmetries will be discussed in more detail in Section 3.1).

It is worth mentioning that the normalization in (31) for the Jost solutions differs from the one considered, for instance, in Refs. [19, 28], where, instead of $W_{ \pm}(k)$, the asymptotic eigenvectors are taken to be

$$
U_{ \pm}(k)=\left(\begin{array}{cc}
\lambda+k & -i q_{ \pm} \\
i q_{ \pm}^{*} & \lambda+k
\end{array}\right) .
$$

The latter choice, however, turned out not to be convenient for the generalization to the vector NLS (cf. [37, 38]), and therefore in the following we will adopt (31) as our normalization for the Jost solutions.

PROPOSITION 3. Suppose the entries of $Q(x)-Q_{f}(x)$ belong to $L^{1}(\mathbb{R})$. Then for each $x \in \mathbb{R}$ the Jost solutions $e^{-i \lambda x} \psi(x, k)$ and $e^{i \lambda x} \phi(x, k)$ are continuous for $k \in \overline{\mathbb{K}^{+}} \backslash\left\{ \pm q_{0}\right\}$ and analytic for $k \in \mathbb{K}^{+}$, while the Jost solutions $e^{i \lambda x} \bar{\psi}(x, k)$ and $e^{-i \lambda x} \bar{\phi}(x, k)$ are continuous for $k \in \overline{\mathbb{K}^{-}} \backslash\left\{ \pm q_{0}\right\}$ and analytic for $k \in \mathbb{K}^{-}$. In addition, if $Q(x)-Q_{f}(x)$ belongs to $L^{1,2}(\mathbb{R})$, then $e^{-i \lambda x} \psi(x, k)$ and $e^{i \lambda x} \phi(x, k)$ are continuous for $k \in \overline{\mathbb{K}^{+}}$and analytic for $k \in \mathbb{K}^{+}$, while the Jost solutions $e^{i \lambda x} \bar{\psi}(x, k)$ and $e^{-i \lambda x} \bar{\phi}(x, k)$ are continuous for $k \in \overline{\mathbb{K}^{-}}$and analytic for $k \in \mathbb{K}^{-}$.

Proof. Let us consider, for $k \in \Sigma \backslash\left\{ \pm q_{0}\right\}$, the matrices $P_{-i \lambda}^{ \pm}$and $P_{i \lambda}^{ \pm}$ defined as follows:

$$
\begin{gathered}
P_{-i \lambda}^{ \pm}(k)=\operatorname{Res}_{\zeta=-i \lambda}\left(\zeta I_{2}-A_{ \pm}(k)\right)^{-1}=\frac{1}{2 \lambda}\left(\begin{array}{cc}
\lambda+k & i q_{ \pm} \\
i q_{ \pm}^{*} & \lambda-k
\end{array}\right) \\
P_{i \lambda}^{ \pm}(k)=\operatorname{Res}_{\zeta=i \lambda}\left(\zeta I_{2}-A_{ \pm}(k)\right)^{-1}=\frac{1}{2 \lambda}\left(\begin{array}{cc}
\lambda-k & -i q_{ \pm} \\
-i q_{ \pm}^{*} & \lambda+k
\end{array}\right) .
\end{gathered}
$$


$P_{-i \lambda}^{ \pm}$and $P_{i \lambda}^{ \pm}$are complementary projections in the sense that

$$
\begin{gathered}
\left(P_{i \lambda}^{ \pm}\right)^{2}=P_{i \lambda}^{ \pm}, \quad\left(P_{-i \lambda}^{ \pm}\right)^{2}=P_{-i \lambda}^{ \pm}, \quad P_{i \lambda}^{ \pm} P_{-i \lambda}^{ \pm}=P_{-i \lambda}^{ \pm} P_{i \lambda}^{ \pm}=0_{2 \times 2}, \\
P_{i \lambda}^{ \pm}+P_{-i \lambda}^{ \pm}=I_{2} .
\end{gathered}
$$

Moreover, they commute with $A_{ \pm}(k)$ and satisfy

$$
A_{ \pm}(k) P_{-i \lambda}^{ \pm}(k)=-i \lambda P_{-i \lambda}^{ \pm}(k), \quad A_{ \pm}(k) P_{i \lambda}^{ \pm}(k)=i \lambda P_{i \lambda}^{ \pm}(k) .
$$

We can also write for any $x \in \mathbb{R}$

$$
e^{x A_{ \pm}(k)}=e^{-i \lambda x} P_{-i \lambda}^{ \pm}(k)+e^{i \lambda x} P_{i \lambda}^{ \pm}(k) .
$$

For $k \in \Sigma \backslash\left\{ \pm q_{0}\right\}$, multiplying (26a) from the right by either column of $W_{+}(k)$, and (26b) by either column of $W_{-}(k)$, we obtain the integral equations

$$
\begin{aligned}
& e^{i \lambda x} \bar{\psi}(x, k) \\
& =\left(\begin{array}{c}
\lambda+k \\
i q_{+}^{*}
\end{array}\right)-\int_{x}^{\infty} d y\left[P_{-i \lambda}^{+}+e^{2 i \lambda(x-y)} P_{i \lambda}^{+}\right]\left[Q(y)-Q_{+}\right] e^{i \lambda y} \bar{\psi}(y, k), \\
& e^{-i \lambda x} \psi(x, k) \\
& =\left(\begin{array}{c}
\lambda-k \\
-i q_{+}^{*}
\end{array}\right)-\int_{x}^{\infty} d y\left[e^{2 i \lambda(y-x)} P_{-i \lambda}^{+}+P_{i \lambda}^{+}\right]\left[Q(y)-Q_{+}\right] e^{-i \lambda y} \psi(y, k), \\
& e^{i \lambda x} \phi(x, k) \\
& =\left(\begin{array}{c}
\lambda+k \\
i q_{-}^{*}
\end{array}\right)+\int_{-\infty}^{x} d y\left[P_{-i \lambda}^{-}+e^{2 i \lambda(x-y)} P_{i \lambda}^{-}\right]\left[Q(y)-Q_{-}\right] e^{i \lambda y} \phi(y, k), \\
& e^{-i \lambda x} \bar{\phi}(x, k) \\
& =\left(\begin{array}{c}
\lambda-k \\
-i q_{-}^{*}
\end{array}\right)+\int_{-\infty}^{x} d y\left[e^{2 i \lambda(y-x)} P_{-i \lambda}^{-}+P_{i \lambda}^{-}\right]\left[Q(y)-Q_{-}\right] e^{-i \lambda y} \bar{\phi}(y, k) .
\end{aligned}
$$

The projections $P_{-i \lambda}^{ \pm}(k)$ and $P_{i \lambda}^{ \pm}(k)$ admit a natural continuation to $k \in \mathbb{K} \backslash\left\{ \pm q_{0}\right\}$ [i.e., to $\lambda \in \mathbb{C} \backslash\{0\}$ ]. Taking into account that these projections are singular matrices, we can prove that for $k \in \Sigma$ their spectral norms are given by

$$
\left\|P_{-i \lambda}^{ \pm}(k)\right\|=\left\|P_{i \lambda}^{ \pm}(k)\right\|=\frac{|\lambda+k|+|\lambda-k|}{2|\lambda|}=\frac{|k|}{|\lambda|}
$$

where we have used $|\lambda+k|+|\lambda-k|=2 \max \{|\lambda|,|k|\}$ for $\lambda, k \in \mathbb{R}$. For $k \notin \Sigma$, we use

$$
\left\|P_{ \pm i \lambda}^{ \pm}\right\|=\sqrt{\frac{1}{4|\lambda|^{2}}\left(|\lambda+k|^{2}+q_{0}^{2}+q_{0}^{2}+|\lambda-k|^{2}\right)}=\frac{\sqrt{2|\lambda|^{2}+2|k|^{2}+2 q_{0}^{2}}}{2|\lambda|} .
$$


Thus the expression $P_{-i \lambda}^{ \pm}(k)+e^{2 i \lambda(x-y)} P_{i \lambda}^{ \pm}(k)$ appearing in (35a) is bounded above in the norm by twice the amount in (36) if $y \geq x$ and $\lambda \in \Lambda^{-} \cup \mathbb{R} \backslash\{0\}$. Iterating (35a) we get

$$
\begin{aligned}
\left\|e^{i \lambda x} \bar{\psi}(x, k)\right\| \leq & \sqrt{|\lambda+k|^{2}+q_{0}^{2}} \\
& +\frac{2|k|}{|\lambda|} \int_{x}^{\infty} d y\left\|Q(y)-Q_{+}\right\|\left\|e^{i \lambda y} \bar{\psi}(y, k)\right\| .
\end{aligned}
$$

Assuming that $Q-Q_{+}$is in $L^{1}$ on any right half-line, which follows from $Q-Q_{ \pm} \in L^{1}\left(\mathbb{R}^{ \pm}\right)$, we see that the series resulting by iteration is absolutely convergent, uniformly in $(x, \lambda)$ on each set of the type

$$
\left\{(x, \lambda): x \geq x_{0}>-\infty,|\lambda| \geq \varepsilon>0, \lambda \in \Lambda^{-} \cup \Sigma\right\} .
$$

Since each term is continuous in $k \in \mathbb{K}^{-} \cup \Sigma \backslash\left\{ \pm q_{0}\right\}$ and analytic in $k \in \mathbb{K}^{-}$ for each $x \in \mathbb{R}$, we arrive at the same continuity and analyticity properties for $e^{i \lambda x} \bar{\psi}(x, k)$.

To extend the continuity properties to $k= \pm q_{0}$, we write the left factor under the integral sign of (35a) as follows:

$$
I_{2}+\left[e^{2 i \lambda(x-y)}-1\right] P_{i \lambda}^{+},
$$

where we have used the complementarity of the projections $P_{ \pm i \lambda}^{+}$. This expression we then estimate in the norm for $k \in \mathbb{K}^{-} \cup \Sigma$ by

$$
\begin{aligned}
1+\left|e^{2 i \lambda(x-y)}-1\right| \frac{|k|}{|\lambda|} & \leq 1+2|x-y||k| \\
& \leq \max (1,2|k|)(1+|x|)(1+|y|) .
\end{aligned}
$$

The proof then proceeds as before, but under the strengthened integrability condition that $Q(x)-Q_{f}(x)$ belongs to $L^{1,2}(\mathbb{R})$. This completes the proof for the Jost solution $\bar{\psi}(x, k)$. The proof for the other three Jost solutions can be carried out in a similar way.

Note that the analyticity domains of the Jost solutions are subsets of only one sheet. Further, the proportionality of the inhomogeneous terms in (35a) and (35b) and in (35c) and (35d) for $k= \pm q_{0}$ implies the proportionality of their solutions, so it is

$$
\psi\left(x, \pm q_{0}\right)=-\bar{\psi}\left(x, \pm q_{0}\right), \quad \phi\left(x, \pm q_{0}\right)=-\bar{\phi}\left(x, \pm q_{0}\right) .
$$

\subsection{Transition and scattering coefficients}

We define below two types of scattering coefficients: the transition coefficients which express the proportionality of the fundamental eigensolutions, and the scattering coefficients which realize the proportionality of the Jost solutions. 
Since $\tilde{\Psi}(x, k)$ and $\tilde{\Phi}(x, k)$ are square matrix solutions of the homogeneous first order system (10), we necessarily have, in view of (12) and (19),

$$
\tilde{\Psi}(x, k)=\tilde{\Phi}(x, k) \mathbb{A}_{l}(k), \quad \tilde{\Phi}(x, k)=\tilde{\Psi}(x, k) \mathbb{A}_{r}(k),
$$

where $\mathbb{A}_{l}(k)$ and $\mathbb{A}_{r}(k)$ are the transition coefficient matrices. In order to decompose $\mathbb{A}_{l}(k)$ and $\mathbb{A}_{r}(k)$ into elements that are analytic in $\lambda \in \Lambda^{+}$and $\lambda \in \Lambda^{-}$, we need to apply the similarity transformation used for the same purpose with the fundamental eigensolutions. As a result of (30) and (38), for $k \in \Sigma$ we get

$$
\begin{aligned}
& (\phi(x, k) \quad \bar{\phi}(x, k))=(\bar{\psi}(x, k) \quad \psi(x, k)) S(k), \\
& (\bar{\psi}(x, k) \quad \psi(x, k))=(\phi(x, k) \quad \bar{\phi}(x, k)) \bar{S}(k),
\end{aligned}
$$

where $[19,28]$

$$
S(k)=W_{+}^{-1}(k) \mathbb{A}_{r}(k) W_{-}(k)=\left(\begin{array}{cc}
a(k) & \bar{b}(k) \\
b(k) & \bar{a}(k)
\end{array}\right),
$$

and $\bar{S}(k)=W_{-}^{-1}(k) \mathbb{A}_{l}(k) W_{+}(k)=S^{-1}(k)$. Comparing the asymptotic behavior of the Jost solutions as $x \rightarrow \pm \infty$ and taking into account (23), yields the following quasi-unitarity relation for the scattering coefficients for $k \in \Sigma \backslash\left\{ \pm q_{0}\right\}:$

$$
a(k) \bar{a}(k)-b(k) \bar{b}(k)=e^{i \Theta},
$$

where $\Theta=\theta_{+}-\theta_{-}$. As a consequence, one also has

$$
\bar{S}(k)=e^{-i \Theta}\left(\begin{array}{cc}
\bar{a}(k) & -\bar{b}(k) \\
-b(k) & a(k)
\end{array}\right) .
$$

As a result of (29), we easily find

$$
\left[D^{1 / 2}(k) S(k) D^{-1 / 2}(k)\right]^{\dagger}=\sigma_{3}\left[D^{1 / 2}(k) S(k) D^{-1 / 2}(k)\right]^{-1} \sigma_{3} .
$$

Observe that (40) becomes singular for $k= \pm q_{0}$, due to the noninvertibility of $W_{ \pm}\left(q_{0}\right)$ and $W_{ \pm}\left(-q_{0}\right)$.

Note that using (20) in (40) and taking into account (30) and (28), we get the following integral representations for the scattering coefficients:

$$
\begin{aligned}
\left(\begin{array}{cc}
a(k) & \bar{b}(k) \\
b(k) & \bar{a}(k)
\end{array}\right)= & \int_{0}^{\infty} d y e^{i \lambda y \sigma_{3}} W_{+}^{-1}(k)\left[Q(y)-Q_{+}\right](\phi(y, k) \bar{\phi}(y, k)) \\
+ & W_{+}^{-1}(k) W_{-}(k)\left[I_{2}+\int_{-\infty}^{0} d y e^{i \lambda y \sigma_{3}} W_{-}^{-1}(k)\right. \\
& \left.\times\left[Q(y)-Q_{-}\right](\phi(y, k) \bar{\phi}(y, k))\right]
\end{aligned}
$$


We can now establish the analyticity properties of the scattering coefficients.

Proposition 4. Suppose the entries of $Q(x)-Q_{f}(x)$ belong to $L^{1}(\mathbb{R})$. Then a $(k)$ is continuous in $k \in \overline{\mathbb{K}^{+}} \backslash\left\{ \pm q_{0}\right\}$ and analytic in $k \in \mathbb{K}^{+}$, while $\bar{a}(k)$ is continuous in $k \in \overline{\mathbb{K}^{-}} \backslash\left\{ \pm q_{0}\right\}$ and analytic in $k \in \mathbb{K}^{-}$. The functions $b(k), \bar{b}(k)$ are continuous in $k \in \Sigma \backslash\left\{ \pm q_{0}\right\}$, but in general cannot be continued off $\Sigma$.

Proof. By using (39) we can represent the scattering coefficients as Wronskians of the Jost solutions:

$$
\begin{array}{ll}
a(k)=\frac{\operatorname{Wr}(\phi(x, k), \psi(x, k))}{\operatorname{Wr}(\bar{\psi}(x, k), \psi(x, k))}, & \bar{a}(k)=-\frac{\operatorname{Wr}(\bar{\phi}(x, k), \bar{\psi}(x, k))}{\operatorname{Wr}(\bar{\psi}(x, k), \psi(x, k))}, \\
b(k)=-\frac{\operatorname{Wr}(\phi(x, k), \bar{\psi}(x, k))}{\operatorname{Wr}(\bar{\psi}(x, k), \psi(x, k))}, & \bar{b}(k)=\frac{\operatorname{Wr}(\bar{\phi}(x, k), \psi(x, k))}{\operatorname{Wr}(\bar{\psi}(x, k), \psi(x, k))} .
\end{array}
$$

Here the Wronskian $\operatorname{Wr}(u, v)$ of the two column vectors $u$ and $v$ is defined as $\operatorname{Wr}(u, v)=u^{T} \sigma_{3} \sigma_{1} v=u^{(1)} v^{(2)}-u^{(2)} v^{(1)}$. Given two column solutions $u(x, k)$ and $v(x, k)$ of Equation (5), it easy to show that $\operatorname{Wr}(u, v)$ does not depend on $x$. As a consequence, from (31a) one has

$$
\operatorname{Wr}(\bar{\psi}(x, k), \psi(x, k))=-2 i \lambda q_{+}^{*}
$$

and therefore

$$
\begin{aligned}
& a(k)=-\frac{\operatorname{Wr}(\phi(x, k), \psi(x, k))}{2 i \lambda q_{+}^{*}}, \quad \bar{a}(k)=\frac{\operatorname{Wr}(\bar{\phi}(x, k), \bar{\psi}(x, k))}{2 i \lambda q_{+}^{*}}, \\
& b(k)=\frac{\operatorname{Wr}(\phi(x, k), \bar{\psi}(x, k))}{2 i \lambda q_{+}^{*}}, \quad \bar{b}(k)=-\frac{\operatorname{Wr}(\bar{\phi}(x, k), \psi(x, k))}{2 i \lambda q_{+}^{*}} .
\end{aligned}
$$

From the analyticity properties of the Jost solutions established in Proposition 3 , and since $\lambda \neq 0$ if $k \neq \pm q_{0}$, the proof follows. We note that the analyticity of the scattering data could also be established by means of the integral representations (44).

With the help of Proposition 3 and Equations (46), we easily prove the following.

Corollary 1. Suppose the entries of $Q(x)-Q_{f}(x)$ belong to $L^{1,2}(\mathbb{R})$. Then $\lambda(k) a(k)$ is analytic in $k \in \mathbb{K}^{+}$and continuous in $k \in \overline{\mathbb{K}^{+}}, \lambda(k) \bar{a}(k)$ is analytic in $k \in \mathbb{K}^{-}$and continuous in $k \in \overline{\mathbb{K}^{-}}$, and $\lambda(k) b(k)$ and $\lambda(k) \bar{b}(k)$ are continuous for $k \in \mathbb{R}$. 
Taking into account the analyticity properties of the Jost solutions, we are interested in determining the $2 \times 2$ matrices

$$
\boldsymbol{T}(k)=\left(\begin{array}{cc}
t_{l}(k) & r(k) \\
\rho(k) & t_{r}(k)
\end{array}\right)
$$

and

$$
\overline{\boldsymbol{T}}(k)=\left(\begin{array}{ll}
\bar{t}_{r}(k) & \bar{\rho}(k) \\
\bar{r}(k) & \bar{t}_{l}(k)
\end{array}\right)
$$

which allow us to formulate the Riemann-Hilbert problems

$$
\begin{aligned}
& (\phi(x, k) \quad \psi(x, k))=(\bar{\psi}(x, k) \quad \bar{\phi}(x, k)) \sigma_{3} \overline{\boldsymbol{T}}(k) \sigma_{3}, \\
& (\bar{\psi}(x, k) \quad \bar{\phi}(x, k))=(\phi(x, k) \quad \psi(x, k)) \sigma_{3} \boldsymbol{T}(k) \sigma_{3},
\end{aligned}
$$

where the scattering coefficients $t_{l}(k), t_{r}(k), \bar{t}_{l}(k), \bar{t}_{r}(k)$ are called the (left and right) "transmission coefficients," while $\rho(k), \bar{\rho}(k), r(k), \bar{r}(k)$ are the (left and right) "reflection coefficients." Note that the matrix $(\phi(x, k) \quad \psi(x, k))$ is analytic for $k \in \mathbb{K}^{+}$and continuous for $k \in \overline{\mathbb{K}^{+}}$, whereas the matrix $(\bar{\psi}(x, k) \bar{\phi}(x, k))$ is analytic for $k \in \mathbb{K}^{-}$and continuous for $k \in \overline{\mathbb{K}^{-}}$. A comparison between (39) and (47) gives us the following expressions for the scattering coefficients:

$$
\begin{aligned}
& t_{l}(k)=1 / a(k), \quad t_{r}(k)=e^{i \Theta} / a(k), \\
& \rho(k)=b(k) / a(k), \quad r(k)=-\bar{b}(k) / a(k), \\
& \bar{t}_{r}(k)=e^{i \Theta} / \bar{a}(k), \quad \bar{t}_{l}(k)=1 / \bar{a}(k), \\
& \bar{\rho}(k)=\bar{b}(k) / \bar{a}(k), \quad \bar{r}(k)=-b(k) / \bar{a}(k) .
\end{aligned}
$$

Using (43), we easily obtain that $D^{1 / 2}(k) \boldsymbol{T}(k) D^{-1 / 2}(k)$ is unitary for $k \in \Sigma \backslash\left\{ \pm q_{0}\right\}$.

\section{Direct problem: $z$ variable}

Before we proceed further discussing the properties of the scattering coefficients and posing and solving the inverse problem, it is convenient to introduce a uniformization variable $z$ (cf. [28]) defined by the conformal mapping:

$$
z=k+\lambda(k),
$$

and inverse mapping given by

$$
k=\frac{1}{2}\left(z+\frac{q_{0}^{2}}{z}\right), \quad \lambda=z-k=\frac{1}{2}\left(z-\frac{q_{0}^{2}}{z}\right) .
$$


We observe that

- the two sheets $\mathbb{K}^{+}, \mathbb{K}^{-}$of the Riemann surface $\mathbb{K}$ are, respectively, mapped onto the upper and lower half-planes $\mathbb{C}^{ \pm}$of the complex $z$-plane;

- the cut $\Sigma$ on the Riemann surface is mapped onto the real $z$ axis;

- the segments $-q_{0} \leq k \leq q_{0}$ on $\mathbb{K}^{+}$and $\mathbb{K}^{-}$are mapped onto the upper and lower semicircles of radius $q_{0}$ and center at the origin of the $z$-plane.

From Proposition 3 it then follows that the Jost solutions $\phi(x, z) e^{i \lambda x}$, $\psi(x, z) e^{-i \lambda x}$ are analytic in the upper half-plane of $z$, while $\bar{\phi}(x, z) e^{-i \lambda x}$, $\bar{\psi}(x, z) e^{i \lambda x}$ are analytic in the lower half-plane. Moreover, by Proposition 4 and Corollary $1, z a(z)$ and $a(z) / z$ are analytic in $z \in \mathbb{C}^{+}$and continuous in $z \in \overline{\mathbb{C}^{+}}, z \bar{a}(z)$ and $\bar{a}(z) / z$ are analytic in $z \in \mathbb{C}^{-}$and continuous in $z \in \overline{\mathbb{C}^{-}}$, and $z b(z), z \bar{b}(z)$ are continuous in $z \in \mathbb{R}$. We will determine the asymptotic behavior of $a(z), \bar{a}(z), b(z)$ and $\bar{b}(z)$ in Section 3.2.

\subsection{Symmetries and discrete eigenvalues}

It is well known that the scattering problem admits two involutions: $(k, \lambda) \rightarrow\left(k^{*}, \lambda^{*}\right)$ and $(k, \lambda) \rightarrow(k,-\lambda)$, or, in terms of the uniformization variable $z: z \rightarrow z^{*}$ and $z \rightarrow q_{0}^{2} / z$. Writing the considerations around (32) in terms of $z$, we obtain the corresponding symmetry relations between the eigenfunctions

$$
\begin{aligned}
& \bar{\phi}(x, z)=-\frac{i q_{-}^{*}}{z} \sigma_{1} \phi^{*}\left(x, z^{*}\right), \quad \psi(x, z)=-\frac{i q_{+}^{*}}{z} \sigma_{1} \bar{\psi}^{*}\left(x, z^{*}\right), \\
& \phi(x, z)=-\bar{\phi}\left(x, q_{0}^{2} / z\right), \quad \bar{\psi}(x, z)=-\psi\left(x, q_{0}^{2} / z\right) .
\end{aligned}
$$

Correspondingly, from (46) (rewritten in terms of the uniform variable) and (49), one can obtain the symmetries of the scattering coefficients:

$$
a(z)=e^{i \Theta} \bar{a}^{*}\left(z^{*}\right), \quad a(z)=\bar{a}\left(q_{0}^{2} / z\right), \quad \operatorname{Im} z \geq 0,
$$

and

$$
b(z)=e^{i \Theta} \frac{z^{2}}{q_{0}^{2}} \bar{b}^{*}(z), \quad b(z)=\bar{b}\left(q_{0}^{2} / z\right), \quad z \in \mathbb{R},
$$

where we recall $\Theta=\theta_{+}-\theta_{-}$. It is also well known [19] that there is a one-to-one correspondence between the poles of the transmission coefficients $t_{l}(z)=1 / a(z)$ and $\bar{t}_{r}(z)=e^{i \Theta} / \bar{a}(z)$ and discrete eigenvalues of the scattering problem (5). Taking into account the symmetries (50), the quasi-unitarity relation (41) becomes:

$$
|a(z)|^{2}-\frac{q_{0}^{2}}{z^{2}}|b(z)|^{2}=1, \quad z \in \mathbb{R} \backslash\left\{ \pm q_{0}\right\} .
$$


Note that the above equation also makes sense for $z \rightarrow 0$, as in Section 3.2 we will show $b(z) / z=o(1 / z)$. Using (51), we see that $a(z) \neq 0$ for $z \in \mathbb{R}$, which means that there are no spectral singularities (sometimes called embedded eigenvalues). Equation (51) also implies

$$
0<\frac{1}{|a(z)|^{2}}=\left|t_{l, r}(z)\right|^{2}=1-\frac{q_{0}^{2}}{z^{2}}|\rho(z)|^{2} .
$$

Note that (51) can also be obtained directly from (23), taking $Z=\tilde{Z}=\left(\begin{array}{ll}\bar{\psi} & \psi\end{array}\right)$ and comparing the limits of $Z^{\dagger} \sigma_{3} Z$ at both space infinities. Equation (51), together with the self-adjointness of the scattering problem, ensure that the transmission coefficients can only have poles at $z=\zeta_{n}=k_{n}+i v_{n}$ and $z=\zeta_{n}^{*}=k_{n}-i v_{n}$, with $-q_{0}<k_{n}<q_{0}$ and $v_{n}=\sqrt{q_{0}^{2}-k_{n}^{2}}>0$. In Ref. [28] it is shown that all poles are simple. In addition, in Appendix B we will prove that if $q-q_{f} \in L^{1,4}(\mathbb{R})$ there is a finite number of poles, all of which belong to the spectral gap $k \in\left(-q_{0}, q_{0}\right)$.

For any pair of eigenvalues $\left\{\zeta_{n}, \zeta_{n}^{*}\right\}_{n=1}^{N}$ on the circle $C_{0}:=\left\{z \in \mathbb{C}:|z|=q_{0}\right\}$ [i.e., for any pair of zeros of $a(z)$ and $\bar{a}(z)$ on the circle $C_{0}$ ], the Wronskian representations (46a) yield:

$$
\phi\left(x, \zeta_{n}\right)=c_{n} \psi\left(x, \zeta_{n}\right), \quad \bar{\phi}\left(x, \zeta_{n}^{*}\right)=\bar{c}_{n} \bar{\psi}\left(x, \zeta_{n}^{*}\right),
$$

for some complex constants $c_{n}, \bar{c}_{n}$, with $c_{n}=\bar{c}_{n}$ due to the symmetries (49) between the Jost solutions. Finally, we mention that even though the Jost solutions are continuous at the branch points $\pm q_{0}$ for the class of potentials in Proposition 3, the scattering coefficients generically have simple poles when $z= \pm q_{0}$. In fact, from (46a) it follows that if $\phi$ and $\psi$ are linearly independent at $z=q_{0}$ or $z=-q_{0}$ (i.e., at $\lambda=0$ ), then $a(z)$ has a singularity of the form

$$
\left.a(z)\right|_{z= \pm q_{0}}=\frac{a_{ \pm}}{\lambda}+O(1), \quad a_{ \pm}=i \frac{\operatorname{Wr}\left(\phi\left(x, \pm q_{0}\right), \psi\left(x, \pm q_{0}\right)\right)}{2 q_{+}^{*}} .
$$

However, it may also happen that $\phi(x, z)$ and $\psi(x, z)$ become linearly dependent at either $z=q_{0}$ or $z=-q_{0}$, or both. In this case, either $a_{+}$or $a_{-}$, or both, vanish, so that $a(z)$ is nonsingular near the corresponding branch point. When this happens, in scattering theory $z=-q_{0}$ or $z=q_{0}$ is called a virtual level [28]. The behavior of $b(z)$ in the neighborhood of $z= \pm q_{0}$ is the same as that of $a(z)$. Indeed, from (37) and (46) it immediately follows that

$$
\left.b(z)\right|_{z= \pm q_{0}}=\frac{a_{ \pm}}{\lambda}+O(1) .
$$

The behavior of $\bar{a}(z)$ and $\bar{b}(z)$ at the branch points simply follows using the symmetries (50). When $a_{ \pm} \neq 0$, we have the asymptotic relations

$$
\left.\rho(z)\right|_{z= \pm q_{0}}=1+o(1),\left.\quad \bar{\rho}(z)\right|_{z= \pm q_{0}}=1+o(1) .
$$


In Appendix B, higher order asymptotic expansions at the branch points will be considered.

\subsection{Asymptotic behavior of eigenfunctions and scattering data}

In order to properly pose and solve the inverse problem, one has to determine the asymptotic behavior of eigenfunctions and scattering data both as $z \rightarrow \infty$ and as $z \rightarrow 0$. Standard Wentzel-Kramers-Brillouin (WKB) expansions in terms of the uniformization variable $z$ in the scattering problem (see Appendix C) yield the following asymptotic behaviors for the eigenfunctions in the upper-half plane (UHP) of $z$ :

- as $z \rightarrow \infty$ :

$$
\phi(x, z) e^{i \lambda x} \sim\left(\begin{array}{c}
z \\
i q^{*}(x)
\end{array}\right), \quad \psi(x, z) e^{-i \lambda x} \sim-\left(\begin{array}{c}
q_{+}^{*} q(x) / z \\
i q_{+}^{*}
\end{array}\right),
$$

- as $z \rightarrow 0$ :

$$
\phi(x, z) e^{i \lambda x} \sim\left(\begin{array}{c}
z q(x) / q_{-} \\
i q_{-}^{*}
\end{array}\right), \quad \psi(x, z) e^{-i \lambda x} \sim-\left(\begin{array}{c}
q_{0}^{2} / z \\
i q^{*}(x)
\end{array}\right) .
$$

Similarly, in the lower-half plane (LHP) of $z$ we obtain:

- as $z \rightarrow \infty$ :

$$
\bar{\phi}(x, z) e^{-i \lambda x} \sim-\left(\begin{array}{c}
q_{-}^{*} q(x) / z \\
i q_{-}^{*}
\end{array}\right), \quad \bar{\psi}(x, z) e^{i \lambda x} \sim\left(\begin{array}{c}
z \\
i q^{*}(x)
\end{array}\right),
$$

- as $z \rightarrow 0$ :

$$
\bar{\phi}(x, z) e^{-i \lambda x} \sim-\left(\begin{array}{c}
q_{0}^{2} / z \\
i q^{*}(x)
\end{array}\right), \quad \bar{\psi}(x, z) e^{i \lambda x} \sim\left(\begin{array}{c}
z q(x) / q_{+} \\
i q_{+}^{*}
\end{array}\right) .
$$

Note that, unlike the expressions given in Ref. [28], the derivation in Appendix $\mathrm{C}$ shows that even the leading order terms of the asymptotic expansions for the eigenfunctions explicitly depend on the potential $q(x)$, not merely on its asymptotic values $q_{ \pm}$. Using the Wronskian representations (46a) for the scattering data for $z$ in the UHP yields:

$$
a(z) \sim 1 \quad \text { as } z \rightarrow \infty, \quad a(z) \sim q_{+} / q_{-} \quad \text { as } z \rightarrow 0,
$$

as well as:

$$
\bar{a}(z) \sim q_{+} / q_{-} \quad \text { as } z \rightarrow \infty, \quad \bar{a}(z) \sim 1 \quad \text { as } z \rightarrow 0,
$$

for $z$ in the LHP. Similarly, the Wronskian representation of $b(z)$ provides its asymptotic behavior for $z \in \mathbb{R}$. Specifically, for $\left(q-q_{ \pm}\right) \in L^{1}\left(\mathbb{R}^{ \pm}\right)$one has:

$$
\lim _{z \rightarrow \infty} z b(z)=0, \quad \lim _{z \rightarrow 0} \frac{b(z)}{z^{2}}=0
$$


and consequently

$$
\lim _{z \rightarrow \infty} z \rho(z)=0, \quad \lim _{z \rightarrow 0} \frac{\rho(z)}{z^{2}}=0
$$

The asymptotic behaviors for $\bar{b}(z)$ and $\bar{\rho}(z)$ can be obtained analogously, or by simply using the symmetry (50b).

\subsection{Trace formula and area theorem}

Taking into account the analyticity properties of $a(z)$ in the UHP, the location of its zeros, as well as (51), one can obtain the following representation (sometimes referred to as trace formula) for $a(z)$ for $\mathrm{z}$ in UHP:

$$
a(z)=\prod_{n=1}^{N}\left(\frac{z-\zeta_{n}}{z-\zeta_{n}^{*}}\right) \exp \left[-\frac{1}{2 \pi i} \int_{-\infty}^{\infty} \frac{\log \left(1-q_{0}^{2}|\rho(\zeta)|^{2} / \zeta^{2}\right)}{\zeta-z} d \zeta\right]
$$

Recalling that, according to (58), $a(z) \sim q_{+} / q_{-}$as $z \rightarrow 0$, we conclude that the potential satisfies

$$
\frac{q_{+}}{q_{-}}=\prod_{n=1}^{N} \frac{\zeta_{n}}{\zeta_{n}^{*}} \exp \left[-\frac{1}{2 \pi i} \int_{-\infty}^{\infty} \frac{\log \left(1-q_{0}^{2}|\rho(\zeta)|^{2} / \zeta^{2}\right)}{\zeta} d \zeta\right]
$$

Note that the argument of the logarithm is positive, due to the unitarity of $D^{1 / 2}(z) \boldsymbol{T}(z) D^{-1 / 2}(z)$ for $z \in \mathbb{R} \backslash\{0\}$ (see (52)). Following Ref. [28], we refer to (62) as $\Theta$-condition, since $q_{+} / q_{-}$gives the asymptotic phase difference $\Theta \equiv \theta_{+}-\theta_{-}$of the potential. In particular, in the reflectionless case one has that the asymptotic phases and the soliton amplitudes and velocities are not independent from each other. Specifically, they are related via the following condition

$$
\frac{q_{+}}{q_{-}}=\prod_{n=1}^{N} \frac{k_{n}+i v_{n}}{k_{n}-i v_{n}}
$$

If one breaks the integral in (62) into $\left(\int_{-\infty}^{-q_{0}}+\int_{-q_{0}}^{0}+\int_{0}^{q_{0}}+\int_{q_{0}}^{+\infty}\right)$ and in the two integrals $\int_{0}^{ \pm q_{0}}$ performs the change of variable $u=q_{0}^{2} / \zeta$, taking into account the two symmetries (50) yielding

$$
\rho(z)=\frac{z^{2}}{q_{0}^{2}} \rho^{*}\left(q_{0}^{2} / z\right)
$$


one obtains

$$
\begin{aligned}
& \int_{-\infty}^{\infty} \frac{\log \left(1-q_{0}^{2}|\rho(\zeta)|^{2} / \zeta^{2}\right)}{\zeta} d \zeta \\
& \quad=2 \int_{-\infty}^{-q_{0}} \frac{\log \left(1-q_{0}^{2}|\rho(\zeta)|^{2} / \zeta^{2}\right)}{\zeta} d \zeta+2 \int_{q_{0}}^{\infty} \frac{\log \left(1-q_{0}^{2}|\rho(\zeta)|^{2} / \zeta^{2}\right)}{\zeta} d \zeta
\end{aligned}
$$

Since $1 \geq 1-\left(\frac{q_{0}^{2}}{\zeta^{2}}|\rho(\zeta)|^{2}\right)>0$, we see that the first term on the RHS is positive and the second term is negative. Since the two integrals on the RHS are not related to each other by any symmetry, this suggests that in general the radiative part of the spectrum yields a nontrivial contribution to the asymptotic phase difference of the potential.

\section{Riemann-Hilbert problem}

In order to formulate the inverse scattering problem as an RHP, one needs a representation of eigenfunctions that are meromorphic in the UHP of $z$ in terms of a combination of eigenfunctions that are meromorphic in the LHP, via suitably defined jump conditions on the real $z$-axis. Explicitly, we can write

$$
\begin{gathered}
\frac{\phi(x, z)}{a(z)} e^{i \lambda x}-\bar{\psi}(x, z) e^{i \lambda x}=\rho(z) e^{2 i \lambda x} \psi(x, z) e^{-i \lambda x}, \\
\frac{\bar{\phi}(x, z)}{\bar{a}(z)} e^{-i \lambda x}-\psi(x, z) e^{-i \lambda x}=\bar{\rho}(z) e^{-2 i \lambda x} \bar{\psi}(x, z) e^{i \lambda x},
\end{gathered}
$$

where the reflection coefficients $\rho(z)$ and $\bar{\rho}(z)$ are defined as in (48). This set of equations will be considered as a matrix RHP on the real $z$-axis, with poles at the zeros of $a(z)$ in the UHP of $z$ and of $\bar{a}(z)$ in the LHP. Below we solve the RHP by reducing it to a linear system of algebraic-integral equations.

From the asymptotic behavior of eigenfunctions and scattering data, in the UHP we have:

$$
\begin{gathered}
\frac{\phi(x, z)}{a(z)} e^{i \lambda x} \sim\left(\begin{array}{c}
z \\
i q^{*}(x)
\end{array}\right) \quad \text { as } \quad z \rightarrow \infty, \\
\frac{\phi(z)}{a(z)} e^{i \lambda x} \sim\left(\begin{array}{c}
z q(x) / q_{+} \\
i q_{+}^{*}
\end{array}\right) \quad \text { as } \quad z \rightarrow 0 .
\end{gathered}
$$


Similarly, in the LHP:

$$
\begin{aligned}
& \frac{\bar{\phi}(x, z)}{\bar{a}(z)} e^{-i \lambda x} \sim-\left(\begin{array}{c}
q_{+}^{*} q(x) / z \\
i q_{+}^{*}
\end{array}\right) \quad \text { as } \quad z \rightarrow \infty, \\
& \frac{\bar{\phi}(x, z)}{\bar{a}(z)} e^{-i \lambda x} \sim-\left(\begin{array}{c}
q_{0}^{2} / z \\
i q^{*}(x)
\end{array}\right) \quad \text { as } \quad z \rightarrow 0 .
\end{aligned}
$$

In order to take into account the behavior of the eigenfunction at $z=0$, it is convenient to rewrite (64) as

$$
\begin{gathered}
\frac{\phi(x, z)}{z a(z)} e^{i \lambda x}-\frac{\bar{\psi}(x, z)}{z} e^{i \lambda x}=\rho(z) e^{2 i \lambda x} \frac{\psi(x, z)}{z} e^{-i \lambda x}, \\
\frac{\bar{\phi}(x, z)}{\bar{a}(z)} e^{-i \lambda x}-\psi(x, z) e^{-i \lambda x}=\bar{\rho}(z) e^{-2 i \lambda x} \bar{\psi}(x, z) e^{i \lambda x},
\end{gathered}
$$

so that the functions will be bounded at infinity, though having an additional pole at $z=0$. Taking into account the asymptotic behaviors summarized above, as well the relationships (53) between the eigenfunctions at the discrete eigenvalues, the above system can be written as:

$$
\begin{aligned}
& {\left[\frac{\phi(x, z)}{z a(z)} e^{i \lambda x}-\left(\begin{array}{l}
1 \\
0
\end{array}\right)-\frac{1}{z}\left(\begin{array}{c}
0 \\
i q_{+}^{*}
\end{array}\right)-\sum_{n=1}^{N} \frac{\phi\left(x, \zeta_{n}\right)}{\left(z-\zeta_{n}\right) \zeta_{n} a^{\prime}\left(\zeta_{n}\right)} e^{-v_{n} x}\right]} \\
& -\left[\frac{\bar{\psi}(x, z)}{z} e^{i \lambda x}-\left(\begin{array}{l}
1 \\
0
\end{array}\right)-\frac{1}{z}\left(\begin{array}{c}
0 \\
i q_{+}^{*}
\end{array}\right)-\sum_{n=1}^{N} \frac{c_{n} \psi\left(x, \zeta_{n}\right)}{\left(z-\zeta_{n}\right) \zeta_{n} a^{\prime}\left(\zeta_{n}\right)} e^{-v_{n} x}\right] \\
& =\rho(z) e^{2 i \lambda x} \frac{\psi(x, z)}{z} e^{-i \lambda x}, \\
& {\left[\frac{\bar{\phi}(x, z)}{\bar{a}(z)} e^{-i \lambda x}-\left(\begin{array}{c}
0 \\
-i q_{+}^{*}
\end{array}\right)-\frac{1}{z}\left(\begin{array}{c}
-q_{0}^{2} \\
0
\end{array}\right)-\sum_{n=1}^{N} \frac{\bar{\phi}\left(x, \zeta_{n}^{*}\right)}{\left(z-\zeta_{n}^{*}\right) \bar{a}^{\prime}\left(\zeta_{n}^{*}\right)} e^{-v_{n} x}\right]} \\
& \left.-\left[\begin{array}{c}
0 \\
\psi
\end{array}\right)-\frac{1}{z}\left(\begin{array}{c}
-q_{0}^{2} \\
0
\end{array}\right)-\sum_{n=1}^{N} \frac{\bar{c}_{n} \bar{\psi}\left(x, \zeta_{n}^{*}\right)}{\left(z-\zeta_{n}^{*}\right) \bar{a}^{\prime}\left(\zeta_{n}^{*}\right)} e^{-v_{n} x}\right] \\
& =\bar{\rho}(z) e^{-i \lambda \lambda x} \bar{\psi}(x, z) e^{i \lambda x},
\end{aligned}
$$

where $a^{\prime}(z), \bar{a}^{\prime}(z)$ denote derivatives with respect to $z$, and we have also used $e^{i \lambda\left(\zeta_{n}\right) x}=e^{-i \lambda\left(\zeta_{n}^{*}\right) x}=e^{-v_{n} x}$. We now introduce the Cauchy projectors:

$$
\Pi_{ \pm}(f)(z)=\frac{1}{2 \pi i} \int_{-\infty}^{+\infty} \frac{f(\zeta)}{\zeta-(z \pm i 0)} d \zeta
$$

which are well defined for any function $f(\zeta)$ that is integrable on the real axis. $\Pi_{ \pm}(f)(z)$ is analytic in the upper/lower half plane and, if $f_{ \pm}$admit analytic 
continuation in the upper/lower half plane, one has:

$$
\Pi_{ \pm}\left(f_{ \pm}\right)(z)= \pm f_{ \pm}(z), \quad \Pi_{\mp}\left(f_{ \pm}\right)(z)=0 .
$$

Applying the projector $\Pi_{-}$to the first equation and $\Pi_{+}$to the second equation, we obtain

$$
\begin{aligned}
\bar{\psi}(x, z) e^{i \lambda x}= & \left(\begin{array}{c}
z \\
i q_{+}^{*}
\end{array}\right)+\sum_{n=1}^{N} \frac{z}{\left(z-\zeta_{n}\right)} C_{n} \psi\left(x, \zeta_{n}\right) e^{-v_{n} x} \\
& -\frac{z}{2 \pi i} \int_{-\infty}^{+\infty} \frac{\rho(\zeta)}{\zeta} \frac{\psi(x, \zeta)}{\zeta-(z-i 0)} e^{i \lambda(\zeta) x} d \zeta, \\
\psi(x, z) e^{-i \lambda x}= & -\left(\begin{array}{c}
q_{0}^{2} / z \\
i q_{+}^{*}
\end{array}\right)+\sum_{n=1}^{N} \frac{\bar{C}_{n}}{\left(z-\zeta_{n}^{*}\right)} \bar{\psi}\left(x, \zeta_{n}^{*}\right) e^{-v_{n} x} \\
& +\frac{1}{2 \pi i} \int_{-\infty}^{+\infty} \frac{\bar{\rho}(\zeta)}{\zeta-(z+i 0)} \bar{\psi}(x, \zeta) e^{-i \lambda(\zeta) x} d \zeta,
\end{aligned}
$$

where we have introduced the norming constants:

$$
C_{n}=\frac{c_{n}}{\zeta_{n} a^{\prime}\left(\zeta_{n}\right)}, \quad \bar{C}_{n}=\frac{\bar{c}_{n}}{\bar{a}^{\prime}\left(\zeta_{n}^{*}\right)} .
$$

Recalling that $\bar{c}_{n}=c_{n}$, the symmetries (50) yield the following constraints for the norming constants:

$$
\bar{C}_{n}=-\zeta_{n}^{*} C_{n}, \quad \bar{C}_{n}^{*}=-\bar{C}_{n} .
$$

The system (67) is closed by evaluating the first equation at $z=\zeta_{n}^{*}$ and the second at $z=\zeta_{n}$.

From (57b), the limit as $z \rightarrow 0$ of the first component of $\bar{\psi}(x, z) e^{i \lambda x}$ gives:

$$
\begin{aligned}
q(x)=q_{+} & {\left[1-\sum_{n=1}^{N} \frac{C_{n}}{\zeta_{n}} \psi^{(1)}\left(x, \zeta_{n}\right) e^{-v_{n} x}\right.} \\
& \left.-\frac{1}{2 \pi i} \int_{-\infty}^{+\infty} \frac{\rho(\zeta)}{\zeta^{2}} \psi^{(1)}(x, \zeta) e^{i \lambda(\zeta) x} d \zeta\right]
\end{aligned}
$$

where $\psi^{(1)}(x, z)$ denotes the first component of the Jost solution $\psi(x, z)$.

In the reflectionless case, i.e., when $\rho(z) \equiv 0$ for all $z \in \mathbb{R}$, the linear system (67) reduces to a linear algebraic system of equations, and the potential is then reconstructed simply as

$$
q(x)=q_{+}\left[1-\sum_{j, n=1}^{N} \frac{\bar{C}_{n}}{\zeta_{n}}\left(A^{-1}(x)\right)_{n, j} e^{-2 v_{n} x}\right]
$$


where $A^{-1}(x)$ is the inverse matrix of $A(x)$, with entries

$$
A_{j, n}(x)=\delta_{j, n}+\frac{\bar{C}_{n}}{\zeta_{j}-\zeta_{n}^{*}} e^{-2 v_{n} x} \quad j, n=1, \ldots, N .
$$

Finally, we mention that the asymptotic behaviors determined in Section 3.2 ensures that the integrals in (67) and (70) are well defined.

\section{Marchenko equations and reconstruction of potentials}

In this section we formulate the inverse problem in terms of the Marchenko equations. We solve explicitly these equations in the reflectionless case $(\rho(z) \equiv 0$ for all $z \in \mathbb{R}$ ) by using a suitable triplet of matrices and matrix exponentials. As a result, we get an explicit compact formula for the representation of the multisoliton solutions to (3).

\subsection{Solving the Marchenko equations}

It is well known (see Equations (7.37) and (7.44) in Chapter 2 of Ref. [28]) that the inverse scattering theory of (5) can be formulated in terms of the Marchenko equations.

Let us introduce the triangular representations of the Jost solutions (cf. [28], with a different normalization) as:

$$
\begin{aligned}
& \bar{\psi}(x, z)=\left[e^{-i \lambda(z) x} I_{2}+\int_{x}^{\infty} d s \boldsymbol{K}(x, s) e^{-i \lambda(z) s}\right] w_{1,+}(z), \\
& \psi(x, z)=\left[e^{i \lambda(z) x} I_{2}+\int_{x}^{\infty} d s \boldsymbol{K}(x, s) e^{i \lambda(z) s}\right] w_{2,+}(z)
\end{aligned}
$$

where $w_{1,+}(z)$ and $w_{2,+}(z)$ denote the column vectors of $W_{+}$in Equation (27), expressed in terms of the uniform variable $z$. Also here $\boldsymbol{K}(x, y)$ is a $2 \times 2$ matrix function which has to satisfy the following Marchenko equation:

$$
\boldsymbol{K}(x, y)+\mathbb{G}(x+y)+\int_{x}^{\infty} d s \boldsymbol{K}(x, s) \mathbb{G}(s+y)=0,
$$

where $\boldsymbol{K}(x, y)$ and $\mathbb{G}(s+y)$ are defined as

$$
\begin{gathered}
\boldsymbol{K}(x, y)=(\bar{K}(x, y) \quad K(x, y))=\left(\begin{array}{ll}
K_{11}(x, y) & K_{12}(x, y) \\
K_{21}(x, y) & K_{22}(x, y)
\end{array}\right), \\
\mathbb{G}(s+y)=\left(\begin{array}{ll}
F_{1}(s+y) & F_{2}^{*}(s+y) \\
F_{2}(s+y) & F_{1}^{*}(s+y)
\end{array}\right),
\end{gathered}
$$


with

$$
\begin{gathered}
F_{1}(x)=F_{1, c}(x)+i F_{2, c}^{\prime}(x)-\frac{\zeta_{n}^{*}}{2} F_{1, d}(x), \\
F_{2}(x)=-i q_{+}^{*}\left[F_{2, c}(x)+\frac{1}{2} F_{1, d}(x)\right],
\end{gathered}
$$

where

$$
\begin{gathered}
F_{1, c}(x)=\frac{1}{2 \pi} \int_{-\infty}^{\infty} d \zeta e^{i \zeta x} \frac{\rho\left(\sqrt{\zeta^{2}+q_{0}^{2}}, \zeta\right)+\rho\left(-\sqrt{\zeta^{2}+q_{0}^{2}}, \zeta\right)}{2} \\
F_{2, c}(x)=\frac{1}{2 \pi} \int_{-\infty}^{\infty} d \zeta e^{i \zeta x} \frac{\rho\left(\sqrt{\zeta^{2}+q_{0}^{2}}, \zeta\right)-\rho\left(-\sqrt{\zeta^{2}+q_{0}^{2}}, \zeta\right)}{2 \sqrt{\zeta^{2}+q_{0}^{2}}} \\
F_{1, d}(x)=-i \sum_{n=1}^{N} C_{n} e^{-v_{n} x}
\end{gathered}
$$

$\zeta_{n}=k_{n}+i v_{n}$ are the discrete eigenvalues and $C_{n}$ are the norming constants introduced in Equation (68).

Observe that the matrices $\boldsymbol{K}(x, y)$ and $\mathbb{G}(x+y)$ both have the following symmetry properties:

$$
\sigma_{1} T \sigma_{1}=T^{*}
$$

In analogy with the method developed in Refs. [32-35], we now solve explicitly the Marchenko equation (74) in the reflectionless case, i.e., when $\rho(z) \equiv 0$ for all $z \in \mathbb{R}$, and a finite number of discrete eigenvalues $\zeta_{n}$ (see Appendix B for more details). In order to do so, let us represent the $2 \times 2$ matrix Marchenko kernel as follows:

$$
\mathbb{G}(z)=\boldsymbol{C} e^{-z A} \boldsymbol{B},
$$

where $\boldsymbol{A}$ is a $p \times p$ matrix having only eigenvalues with positive real part, $\boldsymbol{B}$ is a $p \times 2$ matrix, and $\boldsymbol{C}$ is a $2 \times p$ matrix. We recall that there are many different triplets $(\boldsymbol{A}, \boldsymbol{B}, \boldsymbol{C})$ which lead to the same representation (78) of the $2 \times 2$ matrix kernel $\mathbb{G}(z)$. Among these representations, we point out the so-called minimal representations, where the triplet is such that $\boldsymbol{A}$ has minimal matrix order among all triplets leading to a representation of the same Marchenko kernel (see [40] for more details). Two triplets $(\boldsymbol{A}, \boldsymbol{B}, \boldsymbol{C})$ and $(\tilde{\boldsymbol{A}}, \tilde{\boldsymbol{B}}, \tilde{\boldsymbol{C}})$ leading to minimal representations of the same $2 \times 2$ Marchenko kernel $\mathbb{G}(z)$ are similar, in the sense that there exists a unique invertible matrix $S$ such that

$$
\tilde{A}=S A S^{-1}, \quad \tilde{B}=S B, \quad \tilde{C}=C S^{-1},
$$

(cf. [40, Theorem 19.4]). 
To get explicit solutions of (74), let us consider Equation (78) in which the minimality of the triplet $(\boldsymbol{A}, \boldsymbol{B}, \boldsymbol{C})$ is assumed and $\boldsymbol{A}$ is a $p \times p$ matrix having only eigenvalues with positive real part. Then the Marchenko equation (74) can be written as

$$
\boldsymbol{K}(x, y)=-\left[\boldsymbol{C} e^{-x \boldsymbol{A}}+\boldsymbol{L}(x)\right] e^{-y \boldsymbol{A}} \boldsymbol{B},
$$

where

$$
\boldsymbol{L}(x)=\int_{x}^{\infty} d s \boldsymbol{K}(x, s) \boldsymbol{C} e^{-s \boldsymbol{A}} .
$$

Let $\boldsymbol{P}$ denote the unique solution of the Sylvester equation

$$
\boldsymbol{A P}+\boldsymbol{P} \boldsymbol{A}=\boldsymbol{B C},
$$

i.e., $\boldsymbol{P}=\int_{0}^{\infty} d z e^{-z \boldsymbol{A}} \boldsymbol{B} \boldsymbol{C} e^{-z \boldsymbol{A}}$. Then

$$
\boldsymbol{L}(x)=-\boldsymbol{C} e^{-2 x \boldsymbol{A}} \boldsymbol{P} e^{-x \boldsymbol{A}}\left[I_{p}+e^{-x \boldsymbol{A}} \boldsymbol{P} e^{-x \boldsymbol{A}}\right]^{-1},
$$

where $I_{p}$ denotes the $p \times p$ identity matrix. Consequently,

$$
\boldsymbol{K}(x, y)=-\boldsymbol{C} e^{-x \boldsymbol{A}}\left[I_{p}+e^{-x \boldsymbol{A}} \boldsymbol{P} e^{-x \boldsymbol{A}}\right]^{-1} e^{-y \boldsymbol{A}} \boldsymbol{B},
$$

provided the inverse matrix appearing in this expression exists for each $x \in \mathbb{R}$.

It is well known that the potential (see [19]) can be reconstructed by means of one of the entries of the Marchenko kernel (75a) as follows:

$$
q(x)=q_{+}-2 K_{12}(x, x),
$$

which implies that $q(x) \rightarrow q_{+}$as $x \rightarrow+\infty$. If we write

$$
\boldsymbol{C}=\left(\begin{array}{l}
\boldsymbol{C}^{(1)} \\
\boldsymbol{C}^{(2)}
\end{array}\right), \quad \boldsymbol{B}=\left(\boldsymbol{B}^{(1)} \boldsymbol{B}^{(2)}\right),
$$

where $\boldsymbol{C}^{(1)}$ and $\boldsymbol{C}^{(2)}$ are rows of length $p$ and $\boldsymbol{B}^{(1)}$ and $\boldsymbol{B}^{(2)}$ are columns of length $p$, we get

$$
\begin{aligned}
q(x) & =q_{+}+2 \boldsymbol{C}^{(1)} e^{-x \boldsymbol{A}}\left[I_{p}+e^{-x \boldsymbol{A}} \boldsymbol{P} e^{-x \boldsymbol{A}}\right]^{-1} e^{-x \boldsymbol{A}} \boldsymbol{B}^{(2)} \\
& =q_{+}+2 \boldsymbol{C}^{(1)}\left[\boldsymbol{P}+e^{2 x \boldsymbol{A}}\right]^{-1} \boldsymbol{B}^{(2)}
\end{aligned}
$$

We observe that the above equation yields

$$
q_{-}=q_{+}+2 \boldsymbol{C}^{(1)} \boldsymbol{P}^{-1} \boldsymbol{B}^{(2)}
$$

in the limit $x \rightarrow-\infty$. Of course, this last conclusion requires knowing that $\boldsymbol{P}$ is invertible.

Note that for fixed $x \in \mathbb{R}$, the existence of the inverse $e^{2 x \boldsymbol{A}}+\boldsymbol{P}$ (which appears in (81)) is equivalent to the unique solvability of the Marchenko 
equation. Therefore, the invertibility of $e^{2 x \boldsymbol{A}}+\boldsymbol{P}$ for every $x \in \mathbb{R}$ is equivalent to the existence of a unique reflectionless potential with given bound states and norming constants.

It is also worth stressing that in the previous papers where the method of the triplets $(\boldsymbol{A}, \boldsymbol{B}, \boldsymbol{C})$ is used [32-35], the invertibility of the matrix $\boldsymbol{P}$ (solution of the Sylvester equation) is guaranteed by assuming the minimality of the triplet $(\boldsymbol{A}, \boldsymbol{B}, \boldsymbol{C})$, and that all the eigenvalues of the matrix $\boldsymbol{A}$ have positive real parts. In the present case, this is not true. In fact, it is not difficult to construct examples where the matrix $\boldsymbol{P}$ is not invertible although the triplet $(\boldsymbol{A}, \boldsymbol{B}, \boldsymbol{C})$ is minimal and all the eigenvalues of $\boldsymbol{A}$ have positive real parts. We provide one such example in Appendix D. This example suggests that the formula (81) still makes sense when $\boldsymbol{P}$ is not invertible, but the corresponding solution blows up as $|x| \rightarrow \infty$. Then, in order to have solutions of (3) satisfying the nonvanishing boundary conditions, we have to assume the minimality of the triplet $(\boldsymbol{A}, \boldsymbol{B}, \boldsymbol{C})$, the invertibility of the matrices $e^{2 x \boldsymbol{A}}+\boldsymbol{P}$ and $\boldsymbol{P}$, and the positivity of the real parts of the eigenvalues of the matrix $\boldsymbol{A}$. On the other hand, it is easy to prove the following [cf. Appendix E].

THEOREM 2. If $\boldsymbol{P}$ is an invertible matrix, then $(\boldsymbol{A}, \boldsymbol{B}, \boldsymbol{C})$ is a minimal triplet.

We mention that matrix triplets to obtain NLS solutions also appear in a non-Marchenko context [41-43].

\subsection{Time evolution}

So far we have obtained solutions of (3) (in the reflectionless case) when $t=0$. In order to get time-dependent solutions, one simply has to insert the time evolution of the scattering data, which is well-known in the literature (see, for instance, Equations (25), (26), and (34) in Ref. [19]), in the equations reconstructing the potential, such as (71) or (81). In particular, the discrete eigenvalues $-q_{0}<k_{n}<q_{0}$ are time-independent, while the time dependence of the reflection coefficients and the norming constants in terms of the original variables $(k, \lambda)$ is given by:

$$
\rho(t)=\rho(0) e^{-4 i k \lambda t}, \quad C_{n}(t)=C_{n}(0) e^{4 k_{n} v_{n} t}
$$

Recalling that $\zeta_{n}=k_{n}+i v_{n}$, with $v_{n}=\sqrt{q_{0}^{2}-k_{n}^{2}}>0$, in the reflectionless case, we can write (77) as

$$
F_{1}(x, t)=\frac{i}{2} \sum_{n=1}^{N} C_{n}(t) \zeta_{n}^{*} e^{-v_{n} x}, \quad F_{2}(x, t)=-\frac{q_{+}^{*}}{2} \sum_{n=1}^{N} C_{n}(t) e^{-v_{n} x}
$$


and

$$
\mathbb{G}(x, t)=\frac{1}{2} \sum_{n=1}^{N} e^{-v_{n} x}\left(\begin{array}{cc}
i C_{n}(t) \zeta_{n}^{*} & -q_{+}(t) C_{n}^{*}(t) \\
-q_{+}^{*}(t) C_{n}(t) & -i \zeta_{n} C_{n}^{*}(t)
\end{array}\right)=\boldsymbol{C}(t) e^{-x \boldsymbol{A}} \boldsymbol{B}(t),
$$

where $\boldsymbol{A}=\operatorname{diag}\left(v_{1}, \ldots, v_{N}\right)$,

$$
\begin{gathered}
\boldsymbol{B}(t)=\frac{1}{2}\left(\begin{array}{ccc}
i \zeta_{1}^{*} C_{1}(t) & -q_{+}(t) C_{1}^{*}(t) \\
\vdots & \vdots \\
i \zeta_{N}^{*} C_{N}(t) & -q_{+}(t) C_{N}^{*}(t)
\end{array}\right), \\
\boldsymbol{C}(t)=\left(\begin{array}{ccc}
1 & \cdots & 1 \\
\frac{i \zeta_{1}}{q_{+}(t)} & \cdots & \frac{i \zeta_{N}}{q_{+}(t)}
\end{array}\right) .
\end{gathered}
$$

As a consequence,

$$
\boldsymbol{P}(t)=\int_{0}^{\infty} d x e^{-x \boldsymbol{A}} \boldsymbol{B}(t) \boldsymbol{C}(t) e^{-x \boldsymbol{A}} .
$$

Finally, to obtain solutions $q(x, t)$ of (3) at the generic time $t$ (in the reflectionless case), it suffices to write down (81) using the triplet $(\boldsymbol{A}, \boldsymbol{B}(t), \boldsymbol{C}(t))$ and the matrix $\boldsymbol{P}(t)$, instead of $(\boldsymbol{A}, \boldsymbol{B}, \boldsymbol{C})$ and $\boldsymbol{P}$.

We can derive the explicit expression of the Jost eigenfunctions in terms of the triplet $\boldsymbol{A}, \boldsymbol{B}, \boldsymbol{C}$. Inserting the expression of (80) into (73), we get

$$
\begin{aligned}
& \bar{\psi}(x, z) \\
& =e^{-i \lambda(z) x}\left\{I_{2}+i \boldsymbol{C} e^{-x \boldsymbol{A}}\left[I_{p}+e^{-x \boldsymbol{A}} \boldsymbol{P} e^{-x \boldsymbol{A}}\right]^{-1} e^{-x \boldsymbol{A}}\left(\lambda(z) I_{p}-i \boldsymbol{A}\right)^{-1} \boldsymbol{B}\right\} w_{1,+}(z) \\
& =e^{-i \lambda(z) x}\left\{I_{2}+i \boldsymbol{C}\left[e^{2 x \boldsymbol{A}}+\boldsymbol{P}\right]^{-1}\left(\lambda(z) I_{p}-i \boldsymbol{A}\right)^{-1} \boldsymbol{B}\right\} w_{1,+}(z) \\
& \psi(x, z) \\
& \quad=e^{i \lambda(z) x}\left\{I_{2}-i \boldsymbol{C} e^{-x \boldsymbol{A}}\left[I_{p}+e^{-x \boldsymbol{A}} \boldsymbol{P} e^{-x \boldsymbol{A}}\right]^{-1} e^{-x \boldsymbol{A}}\left(\lambda(z) I_{p}+i \boldsymbol{A}\right)^{-1} \boldsymbol{B}\right\} w_{2,+}(z) \\
& \quad=e^{i \lambda(z) x}\left\{I_{2}-i \boldsymbol{C}\left[e^{2 x \boldsymbol{A}}+\boldsymbol{P}\right]^{-1}\left(\lambda(z) I_{p}+i \boldsymbol{A}\right)^{-1} \boldsymbol{B}\right\} w_{2,+}(z)
\end{aligned}
$$

If we are interested in the temporal evolution of the Jost solutions, it is sufficient to make the following substitutions $(\boldsymbol{A}, \boldsymbol{B}, \boldsymbol{C}, \boldsymbol{P}) \rightarrow(\boldsymbol{A}, \boldsymbol{B}(t), \boldsymbol{C}(t), \boldsymbol{P}(t))$ in (86) where $\boldsymbol{B}(t), \boldsymbol{C}(t), \boldsymbol{P}(t)$ are defined in (83), (84), and (85).

In the one soliton case, the expressions (for $t=0$ ) of the Jost solutions (86) and the potential (81) are obtained choosing the triplet $(\boldsymbol{A}, \boldsymbol{B}, \boldsymbol{C})$ as:

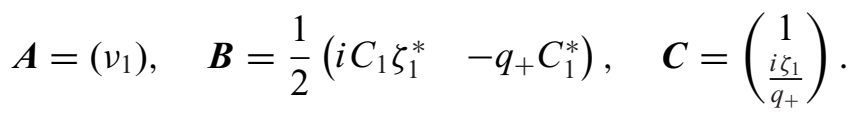


As a result, $\boldsymbol{P}=\left(i C_{1} \zeta_{1}^{*}-i C_{1}^{*} \zeta_{1}\right) /\left(4 v_{1}\right)$. After straightforward calculations and by using the symmetry relations of the norming constants (69), we get

$$
\begin{gathered}
\psi(x, z)=-e^{\frac{i}{2}\left(z-q_{0}^{2} / z\right) x}\left[\left(\begin{array}{c}
q_{0}^{2} / z \\
i q_{+}^{*}
\end{array}\right)-\frac{\bar{C}_{1}}{z-\zeta_{1}^{*}} \frac{e^{-2 v_{1} x}}{1+\frac{\bar{C}_{1}}{\zeta_{1}-\zeta_{1}^{*}} e^{-2 v_{1} x}}\left(\begin{array}{c}
\zeta_{1}^{*} \\
i q_{+}^{*}
\end{array}\right)\right], \\
\bar{\psi}(x, z)=e^{-\frac{i}{2}\left(z-q_{0}^{2} / z\right) x}\left[\left(\begin{array}{c}
z \\
i q_{+}^{*}
\end{array}\right)-\frac{z}{z-\zeta_{1}} \frac{C_{1} e^{-2 v_{1} x}}{1+\frac{\bar{C}_{1}}{\zeta_{1}-\zeta_{1}^{*}} e^{-2 v_{1} x}}\left(\begin{array}{c}
\zeta_{1}^{*} \\
i q_{+}^{*}
\end{array}\right)\right]
\end{gathered}
$$

These expressions are exactly the same one can find solving the linear, algebraic system (67) following from the RHP in the reflectionless case. Moreover, the one soliton solution of (3) is given by:

$$
q(x, t)=q_{+}(t)\left[1-\frac{\bar{C}_{1}(0)}{\zeta_{1}} \frac{e^{-2 v_{1} x+4 k_{1} v_{1} t}}{1+\frac{\bar{C}_{1}(0)}{2 v_{1}} e^{-2 v_{1} x+4 k_{1} v_{1} t}}\right] .
$$

Note that Equation (88) coincides with Equation (2), with $\zeta_{1}=q_{0} e^{i \alpha}$ and $x_{0}=$ $\frac{1}{2 v_{1}} \log \left(\frac{\bar{C}_{1}(0)}{2 v_{1}}\right)$, up to an overall factor $e^{-i \alpha}$.

\section{Acknowledgments}

FD and FV wish to express their gratitude for the hospitality of the Department of Mathematics of the University of Colorado at Colorado Springs where part of the paper was written. BP would like to acknowledge Mark Ablowitz and Gino Biondini for many valuable discussions, some of which during the thematic SQuaRe meetings at the AIM in Palo Alto. The research is partially supported by: (a) NSF under grant no. DMS-10009248; (b) INdAM; (c) MIUR under PRIN 2008 "Geometrical methods in the theory of nonlinear integrable systems" and PRIN grant no. 20083KLJEZ-003; (d) the Autonomous Region of Sardinia (RAS) under grants CRP3-138 and PO Sardegna 2007-2013, L.R. 7/2007.

\section{Appendix A. Fundamental Matrices}

Let us consider the matrices $A_{ \pm}(k)$ introduced in (9a), but allowing $k \in \mathbb{K}$. For $A(x, k)$ defined as in (11) one then has

$$
\beta(k):=\|A(x, k)\|=\left\{\begin{array}{l}
q_{0}+|k|, \quad k \in \mathbb{R} \\
{\left[q_{0}^{2}+|k|^{2}+\sqrt{\left(q_{0}^{2}+|k|^{2}\right)^{2}-|\lambda|^{4}}\right]^{\frac{1}{2}}, \quad k \in \mathbb{K} \backslash \mathbb{R} .}
\end{array}\right.
$$


Consider the initial-value problem

$$
\begin{aligned}
\frac{\partial}{\partial x} \mathcal{G}(x, y ; k) & =A(x, k) \mathcal{G}(x, y ; k), \\
\mathcal{G}(y, y ; k) & =I_{2},
\end{aligned}
$$

where $x, y \in \mathbb{R}$. Then $\mathcal{G}(x, y ; k)$ is defined as a weak solution in the sense that it is the solution of the integral equation

$$
\mathcal{G}(x, y ; k)=I_{2}+\int_{y}^{x} d z A(z, k) \mathcal{G}(z, y ; k) .
$$

Clearly,

$$
\|\mathcal{G}(x, y ; k)\| \leq 1+\beta(k) \int_{\min (x, y)}^{\max (x, y)} d z\|\mathcal{G}(z, y ; k)\|,
$$

so that, by iteration or by Gronwall's inequality,

$$
\|\mathcal{G}(x, y ; k)\| \leq e^{\beta(k)|x-y|} .
$$

This argument also proves the existence of a unique weak solution; this solution exists globally in $(x, y, k) \in \mathbb{R}^{2} \times \mathbb{K}$.

Let us now consider the integral equation

$$
\mathcal{G}^{\prime}(x, y ; k)=I_{2}+\int_{y}^{x} d z \mathcal{G}^{\prime}(x, z ; k) A(z, k) .
$$

We obviously get

$$
\left\|\mathcal{G}^{\prime}(x, y ; k)\right\| \leq 1+\beta(k) \int_{\min (x, y)}^{\max (x, y)} d z\left\|\mathcal{G}^{\prime}(z, y ; k)\right\|,
$$

so that, by iteration or by Gronwall's inequality,

$$
\left\|\mathcal{G}^{\prime}(x, y ; k)\right\| \leq e^{\beta(k)|x-y|} .
$$

Writing down the $n$-th iterates of (A.2) and (A.3) it is immediate to see that they coincide. As a result,

$$
\mathcal{G}(x, y ; k)=\mathcal{G}^{\prime}(x, y ; k) .
$$

Differentiability of $\mathcal{G}(x, y ; k)$ for $x \neq y$ yields the initial-value problem

$$
\begin{gathered}
\frac{\partial}{\partial y} \mathcal{G}(x, y ; k)=-\mathcal{G}(x, y ; k) A(y, k), \\
\mathcal{G}(x, x ; k)=I_{2},
\end{gathered}
$$

where $x, y \in \mathbb{R}$ and $k \in \mathbb{K}$. 
We have the following multiplication property:

$$
\mathcal{G}(x, z ; k) \mathcal{G}(z, y ; k)=\mathcal{G}(x, y ; k)
$$

where $(x, y, z) \in \mathbb{R}^{3}$ and $k \in \mathbb{K}$. Indeed, for fixed $z \in \mathbb{R}$, the matrix function $\mathcal{G}^{\prime \prime}(x, y ; k)=\mathcal{G}(x, z ; k) \mathcal{G}(z, y ; k)$ is easily seen to be a solution of (A.1) and, in the weak sense, this solution is unique. Next, the product rule (A.5) implies the invertibility of $\mathcal{G}(x, y ; k)$, with inverse

$$
\mathcal{G}^{-1}(x, y ; k)=\mathcal{G}(y, x ; k)
$$

The general form of $\mathcal{G}(x, y ; k)$ is easy to find. For $x, y \in \mathbb{R}^{+}$we have $\mathcal{G}(x, y ; k)=e^{(x-y) A_{+}(k)}$; for $x, y \in \mathbb{R}^{-}$we have instead $\mathcal{G}(x, y ; k)=e^{(x-y) A_{-}(k)}$. We then easily get

$$
\mathcal{G}(x, y ; k)= \begin{cases}e^{(x-y) A_{+}(k)}, & x \geq 0, y \geq 0 \\ e^{(x-y) A_{-}(k)}, & x \leq 0, y \leq 0 \\ e^{x A_{+}(k)} e^{-y A_{-}(k)}, & x \geq 0 \geq y \\ e^{x A_{-}(k)} e^{-y A_{-}(k)}, & x \leq 0 \leq y\end{cases}
$$

For each $k \in \mathbb{K}, \mathcal{G}(x, y ; k)$ is Lipschitz continuous in $y$ for fixed $x$ and in $x$ for fixed $y$. As a result, the first order partial derivatives of $\mathcal{G}(x, y ; k)$ exist almost everywhere in the following sense: (a) For fixed $y \in \mathbb{R}, \frac{\partial}{\partial x} \mathcal{G}(x, y ; k)$ exists for almost every $y \in \mathbb{R}$; (b) For fixed $x \in \mathbb{R}, \frac{\partial}{\partial y} \mathcal{G}(x, y ; k)$ exists for almost every $x \in \mathbb{R}$. We can in fact say a bit more. Since $A(x, k)$ is continuous for all $x \in \mathbb{R}$ and differentiable for all $x \in \mathbb{R} \backslash\{0\}$, the two partial derivatives exist in all $(x, y) \in \mathbb{R}^{2}$ where $x \neq y$.

\section{Appendix B: On the Number of Bound States}

In this appendix we identify conditions on the potential that guarantee that there are at most finitely many bound states, all of them belonging to the spectral gap $k \in\left(-q_{0}, q_{0}\right)$. In particular, this allows us to exclude that poles of the transmission coefficient can accumulate at the branch points $\pm q_{0}$.

Let us first prove that the left-hand sides of (35a) and (35d) are differentiable with respect to $\lambda=\lambda(k)$ for every $k \in \overline{\mathbb{K}^{-}} \backslash\{0\}$ if $q-q_{f} \in L^{1,4}(\mathbb{R})$. Similarly, the left-hand sides of (35b) and (35c) are differentiable with respect to $\lambda=\lambda(k)$ for every $k \in \overline{\mathbb{K}^{+}} \backslash\{0\}$ if $q-q_{f} \in L^{1,4}(\mathbb{R})$. Indeed, differentiating 
(35a) with respect to $\lambda$ and using $d k / d \lambda=\lambda / k$, we obtain the integral equation

$$
\begin{aligned}
\frac{\partial}{\partial \lambda} & {\left[e^{i \lambda x} \bar{\psi}(x, \lambda)\right] } \\
= & \left(\begin{array}{c}
\frac{k+\lambda}{k} \\
0
\end{array}\right)-\int_{x}^{\infty} d y F(y-x, \lambda)\left[Q(y)-Q_{+}\right] e^{i \lambda y} \bar{\psi}(y, \lambda) \\
& -\int_{x}^{\infty} d y\left[I_{2}+\left\{e^{2 i \lambda(x-y)}-1\right\} P_{i \lambda}^{+}\right]\left[Q(y)-Q_{+}\right] \frac{\partial}{\partial \lambda}\left[e^{i \lambda y} \bar{\psi}(y, \lambda)\right],
\end{aligned}
$$

where

$$
\begin{aligned}
F(y-x, \lambda)= & \frac{\partial}{\partial \lambda}\left[I_{2}+\left\{e^{2 i \lambda(x-y)}-1\right\} P_{i \lambda}^{+}\right] \\
= & \frac{e^{2 i \lambda(x-y)}-1-2 i \lambda(x-y)}{2 \lambda^{2}}\left(\begin{array}{cc}
\frac{q_{0}^{2}}{k} & i q_{+} \\
i q_{+}^{*} & -\frac{q_{0}^{2}}{k}
\end{array}\right) \\
& +i(x-y)\left\{\left(\begin{array}{cc}
\frac{k-\lambda}{k} & 0 \\
0 & \frac{k+\lambda}{k}
\end{array}\right)+\frac{e^{2 i \lambda(x-y)}-1}{\lambda}\left(\begin{array}{cc}
\lambda-k & -i q_{+} \\
-i q_{+}^{*} & \lambda+k
\end{array}\right)\right\} .
\end{aligned}
$$

Estimating the two fractions containing exponentials in absolute value by $(x-y)^{2}$ and $2|x-y|$, respectively, we see that the inhomogeneous terms in (B.1) are bounded above by $C(1+|x|)^{2}$ if $q-q_{f} \in L^{1,2}(\mathbb{R})$, where the constant $C$ does not depend on $\lambda$ for $\left|k \mp q_{0}\right|<\frac{1}{2} q_{0} .{ }^{1}$ To get the iteration of (B.1) to converge uniformly in $\lambda$ for $\left|k \mp q_{0}\right|<\frac{1}{2} q_{0}$, we replace (B.1) by an inequality by pulling the absolute values under the integral signs and dividing either side by $(1+|x|)^{2}$. Then the iteration converges uniformly in $\lambda$ for $\left|k \mp q_{0}\right|<\frac{1}{2} q_{0}$ and $x$ in bounded real intervals if $q-q_{f} \in L^{1,4}(\mathbb{R})$. A similar argument can be applied to (35b), (35c), and (35d).

Assuming now $q-q_{f} \in L^{1,4}(\mathbb{R})$, we can use an expansion about $\lambda=0$, and obtain as $k \rightarrow \pm q_{0}$ :

$$
\begin{aligned}
\mathrm{Wr}(\phi(x, k), \psi(x, k))= & \operatorname{Wr}\left(\phi\left(x, \pm q_{0}\right), \psi\left(x, \pm q_{0}\right)\right) \\
& +\lambda\left[\frac{\partial}{\partial \lambda} \operatorname{Wr}(\phi(x, k), \psi(x, k)]_{k= \pm q_{0}}+o(\lambda) .\right.
\end{aligned}
$$

As a result of (46) we get

$$
a(k)=\frac{a_{ \pm}}{\lambda}+\alpha_{ \pm}+o(1), \quad k \rightarrow \pm q_{0} .
$$

In the same way we prove that as $k \rightarrow \pm q_{0}$

$$
b(k)=\frac{b_{ \pm}}{\lambda}+\beta_{ \pm}+o(1),
$$

\footnotetext{
${ }^{1}$ This neighborhood of the branch points is chosen small enough to exclude $k=0$, to avoid trouble with the factors $k$ in the denominators.
} 


$$
\begin{aligned}
& \bar{a}(k)=\frac{\bar{a}_{ \pm}}{\lambda}+\bar{\alpha}_{ \pm}+o(1), \\
& \bar{b}(k)=\frac{\bar{b}_{ \pm}}{\lambda}+\bar{\beta}_{ \pm}+o(1) .
\end{aligned}
$$

From (54), (55), and (50) it is clear that $a_{ \pm}=b_{ \pm}$and $\bar{a}_{ \pm}=\bar{b}_{ \pm}$.

PROPOSITION 5. If $q-q_{f} \in L^{1,4}(\mathbb{R})$ the $2 \times 2$ transition matrices $\boldsymbol{T}(k)$ and $\overline{\boldsymbol{T}}(k)$ are continuous for $k \in \Sigma$.

Proof. Let us consider the asymptotic expansions (B.2) and start off by assuming $a_{ \pm} \neq 0$ [then $\bar{a}_{ \pm} \neq 0$, according to (50a)]. From the definitions (48) it then follows

$$
\boldsymbol{T}(k)=\left(\begin{array}{cc}
\frac{\lambda}{a_{ \pm}}\left[1-\frac{\alpha_{ \pm}}{a_{ \pm}} \lambda+o(\lambda)\right] & \frac{\bar{b}_{ \pm}}{a_{ \pm}}\left[1+\lambda\left(\frac{\bar{\beta}_{ \pm}}{b_{ \pm}}-\frac{\alpha_{ \pm}}{a_{ \pm}}\right)+o(\lambda)\right] \\
-\frac{b_{ \pm}}{a_{ \pm}}\left[1+\lambda\left(\frac{\beta_{ \pm}}{b_{ \pm}}-\frac{\alpha_{ \pm}}{a_{ \pm}}\right)+o(\lambda)\right] & \frac{\lambda e^{i \Theta}}{a_{ \pm}}\left[1-\frac{\alpha_{ \pm}}{a_{ \pm}} \lambda+o(\lambda)\right]
\end{array}\right),
$$

and similarly for $\overline{\boldsymbol{T}}(k)$. On the other hand, if $a_{ \pm}=0$ and $\alpha_{ \pm} \neq 0$, we have $b_{ \pm}=\bar{a}_{ \pm}=\bar{b}_{ \pm}=0$ and $\bar{\alpha}_{ \pm} \neq 0$ [cf. (50a), (54), and (55)]. It is then easily verified that

$$
\boldsymbol{T}(k)=\left(\begin{array}{cc}
\frac{1}{\alpha_{ \pm}}+o(1) & -\frac{\bar{\beta}_{ \pm}}{\alpha_{ \pm}}+o(1) \\
\frac{\beta_{ \pm}}{\alpha_{ \pm}}+o(1) & \frac{e^{i \Theta}}{\alpha_{ \pm}}+o(1)
\end{array}\right)
$$

and similarly for $\overline{\boldsymbol{T}}(k)$.

To prove that the case $a_{ \pm}=\alpha_{ \pm}=0$ cannot occur, we now observe that for $a_{ \pm}=0$

$$
a(k)=-\frac{\operatorname{Wr}(\phi(x, k), \psi(x, k))}{2 i \lambda q_{+}^{*}} \rightarrow\left[-\frac{\frac{\partial}{\partial \lambda} \operatorname{Wr}(\phi(x, k), \psi(x, k))}{2 i q_{+}^{*}}\right]_{\lambda=0},
$$

as $k \rightarrow \pm q_{0}$ from within $\Sigma$. Since $t_{l}(k)=(1 / a(k))$ is the $(1,1)$-element of the unitary $2 \times 2$ matrix $D^{1 / 2}(k) \boldsymbol{T}(k) D^{-1 / 2}(k)$, the limit in (B.3) must be $\geq 1$ in absolute value and hence cannot vanish. As a result, $a_{ \pm}$and $\alpha_{ \pm}$cannot vanish simultaneously.

Under the hypothesis of Proposition 5, the number of bound states is finite. This is easily understood as follows: If their number were not finite, then there would be a sequence $\left\{k_{n}\right\}_{n=1}^{\infty}$ of bound states converging to one of the branch points, $q_{0}$ say. In other words, there would exist a sequence $\left\{k_{n}\right\}_{n=1}^{\infty}$ in $\left(-q_{0}, q_{0}\right)$ converging to $q_{0}$ such that $a\left(k_{n}\right)=0(n=1,2,3, \ldots)$. In that case the diagonal elements of $\boldsymbol{T}(k)$ become infinite in absolute value as $k \rightarrow q_{0}$, which contradicts the conclusions of Proposition 5. 


\section{Appendix C: The WKB Expansion of the Jost Solutions}

In the following we will determine the asymptotic behavior of the eigenfunctions as $z \rightarrow 0$ and as $z \rightarrow \infty$ in the proper half-plane. We will derive the WKB expansions for the eigenfunction

$$
N(x, z)=\psi(x, z) e^{-i \lambda(z) x}
$$

in the UHP of $z$. The expansions of the other Jost solutions can be obtained in a similar way.

Based on the boundary condition (31a), we consider the following ansatz for the expansion of the eigenfunction $N(x, z)$ as $z \rightarrow \infty$ :

$$
N(x, z)=\sum_{j=0}^{n} \frac{1}{z^{j}} N_{\infty}^{(j)}(x)+O\left(z^{-(n+1)}\right), \quad N_{\infty}^{(j)}(x)=\left(\begin{array}{l}
N_{\infty}^{1,(j)}(x) \\
N_{\infty}^{2,(j)}(x)
\end{array}\right) .
$$

Substituting these expressions into the scattering problem (5) written in the uniformization variable $z$ and matching the $O(1)$ terms yields:

$$
N_{\infty}^{1,(0)}(x)=0, \quad N_{\infty}^{2,(0)}(x)=-i q_{+}^{*},
$$

where the constant value of $N_{\infty}^{2,(0)}(x)$ is fixed by the asymptotic behavior (31a). The other coefficients are then determined recursively by matching the terms with the same order of $z^{-j}$ :

$$
\begin{gathered}
N_{\infty}^{1,(j)}(x)=i \partial_{x} N_{\infty}^{1,(j-1)}(x)-i q(x) N_{\infty}^{2,(j-1)}(x) \\
\partial_{x} N_{\infty}^{2,(j)}(x)=i q^{*}(x) \partial_{x} N_{\infty}^{1,(j-1)}(x)+i\left(q_{0}^{2}-|q(x)|^{2}\right) N_{\infty}^{2,(j-1)}(x) .
\end{gathered}
$$

For instance, from (C.3) with $j=1$, we have:

$$
N_{\infty}^{1,(1)}(x)=-q_{+}^{*} q(x), \quad N_{\infty}^{2,(1)}(x)=-q_{+}^{*} I_{0}(x),
$$

where

$$
I_{0}(x)=\int_{x}^{+\infty}\left(q_{0}^{2}-\left|q\left(x^{\prime}\right)\right|^{2}\right) d x^{\prime} .
$$

and in the second of (C.4) the constant of integration has been put zero, again based on the asymptotic behavior (31a).

The recurrence relations (C.3) with $j=2$ give:

$$
\begin{aligned}
& N_{\infty}^{1,(2)}(x)=i q_{+}^{*}\left[q(x) I_{0}(x)-\partial_{x} q(x)\right], \\
& N_{\infty}^{2,(2)}(x)=i q_{+}^{*}\left[\frac{1}{2}\left(I_{0}(x)\right)^{2}+I_{1}(x)\right],
\end{aligned}
$$

where

$$
I_{1}(x)=\int_{x}^{+\infty} q^{*}\left(x^{\prime}\right) \partial_{x^{\prime}} q\left(x^{\prime}\right) d x^{\prime}
$$


and so on and so forth. Note that, at each step, solving (C.3b) involves an integration from $x$ to $+\infty$, which then requires stronger integrability conditions on the right half-line for $q-q_{+}$and its $x$-derivatives.

Similarly, we can obtain the asymptotic expansion of $N(x, z)$ as $z \rightarrow 0$ putting

$$
N(x, z)=\sum_{j=-1}^{n} z^{j} N_{0}^{(j)}(x)+O\left(z^{n+1}\right), \quad N_{0}^{(j)}(x)=\left(\begin{array}{l}
N_{0}^{1,(j)}(x) \\
N_{0}^{2,(j)}(x)
\end{array}\right) .
$$

Proceeding as before, we get the following recurrence relations

$$
\begin{gathered}
N_{0}^{2,(j)}(x)=\frac{i}{q_{0}^{2}}\left[q^{*}(x) N_{0}^{1,(j-1)}(x)-\partial_{x} N_{0}^{2,(j-1)}(x)\right], \\
\partial_{x} N_{0}^{1,(j)}(x)=\frac{i}{q_{0}^{2}}\left[\left(|q(x)|^{2}-q_{0}^{2}\right) N_{0}^{1,(j-1)}(x)-q(x) \partial_{x} N_{0}^{2,(j-1)}(x)\right],
\end{gathered}
$$

and the recursion anchored at

$$
N_{0}^{1,(-1)}(x)=-q_{0}^{2}, \quad N_{0}^{2,(-1)}(x)=0 .
$$

For instance, from (C.9) with $j=0$, 1, we have:

$$
\begin{aligned}
& N_{0}^{2,(0)}(x)=-i q^{*}(x), \quad N_{0}^{1,(0)}(x)=-i I_{0}, \\
& N_{0}^{2,(1)}(x)=\frac{1}{q_{0}^{2}}\left[q^{*}(x) I_{0}(x)-\partial_{x} q^{*}(x)\right], \\
& N_{0}^{1,(1)}(x)=\frac{1}{q_{0}^{2}}\left[\frac{1}{2}\left(I_{0}(x)\right)^{2}+I_{1}^{*}(x)\right],
\end{aligned}
$$

and so on and so forth.

\section{Appendix D: On the Noninvertibility of $P$}

In this Appendix we discuss one example in which the triplet $(\boldsymbol{A}, \boldsymbol{B}, \boldsymbol{C})$ is minimal and the eigenvalues of $\boldsymbol{A}$ have positive real parts, but the matrix $\boldsymbol{P}$ satisfying the corresponding Sylvester equation is not invertible.

Consider the matrix triplet $(\boldsymbol{A}, \boldsymbol{B}, \boldsymbol{C})$, where

$$
\boldsymbol{A}=\left(\begin{array}{cc}
2 & i \\
-i & 2
\end{array}\right), \quad \boldsymbol{B}=\left(\begin{array}{cc}
4 & 4+2 i \\
4-2 i & 4
\end{array}\right), \quad \boldsymbol{C}=I_{2},
$$

where $I_{2}$ is the $2 \times 2$ identity matrix. Then $\boldsymbol{A}, \boldsymbol{B}$, and $\boldsymbol{C}$ satisfy the symmetry conditions

$$
\boldsymbol{A}^{*}=\sigma_{1} \boldsymbol{A} \sigma_{1}, \quad \boldsymbol{B}^{*}=\sigma_{1} \boldsymbol{B} \sigma_{1}, \quad \boldsymbol{C}^{*}=\sigma_{1} \boldsymbol{C} \sigma_{1},
$$


where $T^{*}$ is the complex conjugate of the matrix $T$. Then $\boldsymbol{A}$ has the eigenvalues 1 and 3 and the triplet $(\boldsymbol{A}, \boldsymbol{B}, \boldsymbol{C})$ is minimal. In fact,

$$
\begin{aligned}
\mathbb{G}(x) & =\boldsymbol{C} e^{-x \boldsymbol{A}} \boldsymbol{B} \\
& =e^{-2 x}\left(\begin{array}{cc}
4 \cosh (x)-(2+4 i) \sinh (x) & (4+2 i) \cosh (x)-4 i \sinh (x) \\
(4-2 i) \cosh (x)+4 i \sinh (x) & 4 \cosh (x)-(2-4 i) \sinh (x)
\end{array}\right) .
\end{aligned}
$$

It is easily verified that the Sylvester equation $\boldsymbol{A P}+\boldsymbol{P} \boldsymbol{A}=\boldsymbol{B} \boldsymbol{C}$ has the unique solution

$$
\boldsymbol{P}=\sigma_{1} \boldsymbol{P}^{*} \sigma_{1}=\sigma_{1}+I_{2} .
$$

This matrix $\boldsymbol{P}$ is NOT invertible, in spite of the minimality of the triplet $(\boldsymbol{A}, \boldsymbol{B}, \boldsymbol{C})$. Also,

$$
\begin{aligned}
e^{2 x \boldsymbol{A}}+\boldsymbol{P} & =\left(\begin{array}{cc}
1+e^{4 x} \cosh (2 x) & 1+i e^{4 x} \sinh (2 x) \\
1-i e^{4 x} \sinh (2 x) & 1+e^{4 x} \cosh (2 x)
\end{array}\right), \\
{\left[e^{2 x \boldsymbol{A}}+\boldsymbol{P}\right]^{-1} } & =\frac{e^{-8 x}}{1+2 e^{-4 x} \cosh (2 x)}\left(\begin{array}{cc}
1+e^{4 x} \cosh (2 x) & -1-i e^{4 x} \sinh (2 x) \\
-1+i e^{4 x} \sinh (2 x) & 1+e^{4 x} \cosh (2 x)
\end{array}\right), \\
\operatorname{det}\left[e^{2 x \boldsymbol{A}}+\boldsymbol{P}\right] & =e^{8 x}\left[1+2 e^{-4 x} \cosh (2 x)\right] .
\end{aligned}
$$

Consequently, letting $\boldsymbol{C}^{(1)}$ be the first row of $\boldsymbol{C}=I_{2}$ and $\boldsymbol{B}^{(2)}$ the second column of $\boldsymbol{B}$, we get

$$
\begin{aligned}
q(x) & =q_{+}+2 \boldsymbol{C}^{(1)}\left[e^{2 x \boldsymbol{A}}+\boldsymbol{P}\right]^{-1} \boldsymbol{B}^{(2)} \\
& =q_{+}+4 i e^{-2 x} \frac{1-\left(\frac{1}{2}+i\right) e^{6 x}+\left(\frac{3}{2}-i\right) e^{2 x}}{1+e^{4 x}+e^{6 x}},
\end{aligned}
$$

which tends to $q_{+}$as $x \rightarrow+\infty$ and blows up as $x \rightarrow-\infty$.

We conclude giving a simple condition of noninvertibility for the matrix $\boldsymbol{P}$ when the matrix $\boldsymbol{B} \boldsymbol{C}$ has rank one. In fact, if $\boldsymbol{B} \boldsymbol{C}$ has rank one, writing $\boldsymbol{B} \boldsymbol{C}=\boldsymbol{b} \boldsymbol{c}^{T}$ for suitable column vectors $\boldsymbol{b}$ and $\boldsymbol{c}$ and letting $\boldsymbol{A}=\operatorname{diag}\left(a_{1}, \ldots, a_{p}\right)$ for distinct numbers $a_{1}, \ldots, a_{p}$ in the right-hand plane, then [43] (see also [45, Ex. IQ.7.3])

$$
\begin{aligned}
P_{j l} & =\frac{b_{j} c_{l}}{a_{j}+a_{l}}, \\
\operatorname{det} \boldsymbol{P} & =b_{1} \ldots b_{p} c_{1} \ldots c_{p} \operatorname{det}\left(\frac{1}{a_{j}+a_{l}}\right)_{j, l=1}^{p} \\
& =b_{1} \ldots b_{p} c_{1} \ldots c_{p} \prod_{j=1}^{p} \frac{1}{2 a_{j}} \prod_{s<l}\left(\frac{a_{s}-a_{l}}{a_{s}+a_{l}}\right)^{2} .
\end{aligned}
$$

Thus det $\boldsymbol{P}$ is nonzero iff none of the $2 p$ entries of the column vectors $\boldsymbol{b}$ and $\boldsymbol{c}$ vanishes (i.e., iff $\boldsymbol{B} \boldsymbol{C}$ does not have zero rows or zero columns). The results in 
[43], with the exact value of $\operatorname{det} \boldsymbol{P}$, extend to the situation where $\boldsymbol{A}$ is in Jordan normal form, $\boldsymbol{A}$ and $-\boldsymbol{A}$ do not have eigenvalues in common, and $\boldsymbol{B} \boldsymbol{C}$ has rank one. There are no known results if $\boldsymbol{B} \boldsymbol{C}$ has rank $\geq 2$.

\section{Appendix E: Proof of Theorem 2}

If $(\boldsymbol{A}, \boldsymbol{B}, \boldsymbol{C})$ is not a minimal triplet, there exists a minimal triplet $(\tilde{\boldsymbol{A}}, \tilde{\boldsymbol{B}}, \tilde{\boldsymbol{C}})$ such that:

$$
\begin{gathered}
\mathbb{G}(x)=\boldsymbol{C} e^{-x \boldsymbol{A}} \boldsymbol{B}=\tilde{\boldsymbol{C}} e^{-x \tilde{\boldsymbol{A}} \tilde{\boldsymbol{B}},}, \quad x \in \mathbb{R}, \\
\boldsymbol{A}=\left(\begin{array}{ccc}
A_{11} & A_{12} & A_{13} \\
0 & \tilde{\boldsymbol{A}} & A_{23} \\
0 & 0 & A_{33}
\end{array}\right), \quad \boldsymbol{B}=\left(\begin{array}{c}
B_{1} \\
\tilde{\boldsymbol{B}} \\
0
\end{array}\right), \quad \boldsymbol{C}=\left(\begin{array}{lll}
0 & \tilde{\boldsymbol{C}} & C_{3}
\end{array}\right),
\end{gathered}
$$

where $A_{11}$ and $A_{33}$ (and hence $\boldsymbol{A}$ ) have only eigenvalues with positive real parts. Let us write the solution $\tilde{\boldsymbol{P}}$ of the Sylvester equation $\tilde{\boldsymbol{A}} \tilde{\boldsymbol{P}}+\tilde{\boldsymbol{P}} \tilde{\boldsymbol{A}}=\tilde{\boldsymbol{B}} \tilde{\boldsymbol{C}}$ in the form

$$
\boldsymbol{P}=\left(\begin{array}{ccc}
P_{11} & P_{12} & P_{13} \\
0 & \tilde{\boldsymbol{P}} & P_{23} \\
0 & 0 & P_{33}
\end{array}\right)
$$

Then, $\boldsymbol{A} \boldsymbol{P}+\boldsymbol{P} \boldsymbol{A}=\boldsymbol{B} \boldsymbol{C}$ implies

$$
\begin{aligned}
A_{11} P_{11}+P_{11} A_{11} & =0, \\
A_{33} P_{33}+P_{33} A_{33} & =0, \\
P_{12} \tilde{\boldsymbol{A}}+A_{11} P_{12} & =B_{1} \tilde{\boldsymbol{C}}-A_{12} \tilde{\boldsymbol{P}} \\
P_{23} A_{33}+\tilde{\boldsymbol{A}} P_{23} & =\tilde{\boldsymbol{B}} C_{3}-\tilde{\boldsymbol{P}} A_{23}, \\
P_{13} A_{33}+A_{11} P_{13} & =B_{1} C_{3}-P_{12} A_{23}-A_{12} P_{23} .
\end{aligned}
$$

We now use that the Sylvester equation $\mathbb{A}_{1} P+P \mathbb{A}_{2}=Z$ has a unique solution $P$ for each right-hand side $Z$ iff the matrices $\mathbb{A}_{1}$ and $-\mathbb{A}_{2}$ do not have eigenvalues in common [40, Theorem 18.5]. Thus, $P_{11}=0$ and $P_{33}=0$, while there exist unique solutions $P_{12}, P_{23}$, and $P_{13}$. Hence,

$$
\boldsymbol{P}=\left(\begin{array}{ccc}
0 & P_{12} & P_{13} \\
0 & \tilde{\boldsymbol{P}} & P_{23} \\
0 & 0 & 0
\end{array}\right)
$$

which is not invertible. 


\section{References}

1. V. L. GinZBurg, On the theory of superconductivity, Il Nuovo Cimento 2(6):1234-1250 (1955).

2. V. L. Ginzburg and L. D. Landau, Concerning the theory of superconductivity, Sov. Phys. JETP 20:1064-1082 (1958).

3. V. L. Ginzburg and L. P. Pitaevskit, On the theory of superfluidity, Sov. Phys. JETP 34:858-861 (1958).

4. R. Y. Chiao, E. Garmire, and C. H. Townes, Self-trapping of optical beams, Phys. Rev. Lett. 13:479-482 (1964).

5. V. I. TalanOv, About self-focusing of light in cubic media, JETP Lett. 2:138-142 (1965).

6. V. I. TALANOV, On self-focusing of the wave beams in nonlinear media, JEPT Lett. 11: 199-201 (1970).

7. M. J. Ablowitz and H. Segur, Solitons and the Inverse Scattering Transform, SIAM, Philadelphia, 1981.

8. V. E. ZAKHAROV and A. B. SHABAT, Exact theory of two-dimensional self-focusing and one dimensional self-modulation of waves in nonlinear media, Sov. Phys. JETP 34: 62-69 (1972).

9. V. E. Zakharov, Hamilton formalism for hydrodynamic plasma models, Sov. Phys. JETP 33:927-932 (1971); translated from Ž. Ėksper. Teoret. Fiz. 60:1714-1726 (1971) [Russian].

10. A. Hasegawa and F. TAPPeRT, Transmission of stationary nonlinear optical pulses in dispersive dielectric fibers. I. Anomalous dispersion, App. Phys. Lett. 23(3):142-144 (1973).

11. A. Hasegawa and F. TAPPert, Transmission of stationary nonlinear optical pulses in dispersive dielectric fibers. II. Normal dispersion, App. Phys. Lett. 23(3):171-172 (1973).

12. M. Chen, M. A. Tsankov, J. M. Nash, and C. E. Patton, Backward-volume-wave microwave-envelope solitons in yttrium iron garnet films, Phys. Rev. B 49:12773-12790 (1994).

13. A. K. Zvezdin and A. F. Popkov, Contribution to the nonlinear theory of magnetostatic spin waves, Sov. Phys. JETP 57:350-355 (1983).

14. C. J. Pethick and H. Sмith, Bose-Einstein Condensation in Dilute Gases, Cambridge University Press, Cambridge, 2002.

15. M. J. Ablowitz, D. J. Kaup, A. C. Newell, and H. Segur, The Inverse scattering transform. Fourier analysis for nonlinear problems, Stud. Appl. Math. 53:249-315 (1974).

16. M. J. Ablowitz, B. Prinari, and A. D. Trubatch, Discrete and Continuous Nonlinear Schrödinger Systems, London Mathematical Society Lecture Notes Series 302, Cambridge University Press, Cambridge, 2004.

17. F. Calogero and A. Degasperis, Spectral transforms and solitons, North-Holland, Amsterdam, 1982.

18. S. P. Novikov, S. V. Manakov, L. B. Pitaevskit, and V. E. Zakharov, Theory of Solitons: The Inverse Scattering Method, Plenum Press, New York, 1984.

19. V. E. Zakharov and A. B. Shabat, Interaction between solitons in a stable medium, Sov. Phys. JETP 37:823-828 (1973).

20. N. AsANO and Y. KATO, Non-self-adjoint Zakharov-Shabat operator with a potential of the finite asymptotic values, I. Direct and inverse scattering problems, J. Math. Phys. 22:2780-2793 (1980). 
21. N. AsANO and Y. KATO, Non-self-adjoint Zakharov-Shabat operator with a potential of the finite asymptotic values, II. Inverse problem, J. Math. Phys. 25:570-588 (1984).

22. M. Boiti and F. Pempinelli, The spectral transform for the NLS equation with left-right asymmetric boundary conditions, Nuovo Cimento A 69:213-227 (1982).

23. V. S. Gerdikov and P. P. Kulish, Completely integrable Hamiltonian systems connected with a nonselfadjoint Dirac operator, Bulgar. J. Phys. 5(4):337-348 (1978) [Russian].

24. T. Kawata and H. Inoue, Eigenvalue problem with nonvanishing potentials, J. Phys. Soc. Japan 43:361-362 (1977).

25. T. KAWATA and H. INOUE, Inverse scattering method for the nonlinear evolution equations under nonvanishing conditions, J. Phys. Soc. Japan 44:1722-1729 (1978).

26. P. P. Kulish, S. V. Manakov, and L. D. FaddeEv, Comparison of the exact quantum and quasiclassical results for a nonlinear Schrödinger equation, Theor. Math. Phys. 28:615-620 (1976).

27. J. Leon, The Dirac inverse spectral transform: kinks and boomerons, J. Math. Phys. 21(10):2572-2578 (1980).

28. L. D. Faddeev and L. A. TAkhtajan, Hamiltonian Methods in the Theory of Solitons, Springer, Berlin and New York, 1987.

29. V. S. GERDJIKOV and P. P. KULISH, The multicomponent nonlinear Schrödinger equation in the case of nonzero boundary condition, J. Soviet Math. 30:2261-2269 (1985). Also: Zap. Nauchn. Sem. Leningrad. Otdel. Mat. Inst. Steklov. (LOMI) 131:34-46 (1983) [Russian].

30. M. Klaus and J. K. Shaw, On the eigenvalues of the Zakharov-Shabat system, SIAM J. Math. Anal. 34:759-773 (2003).

31. M. KlaUs and C. VAN DER MeE, Wave operators for the matrix Zakharov-Shabat system, J. Math. Phys. 51:053503 (26 pp.) (2010).

32. T. Aktosun, F. Demontis, and C. van der Mee, Exact solutions to the focusing nonlinear Schrödinger equation, Inverse Problems 23:2171-2195 (2007).

33. T. Aktosun, F. Demontis, and C. van Der Mee, Exact solutions to the sine-Gordon equation, J. Math. Phys. 51:123521 (27 pp.) (2010).

34. T. Aktosun and C. VAN DER Mee, Explicit solutions to the Korteweg-de Vries equation on the half line, Inverse Problems 22:2165-2174 (2006).

35. F. Demontis and C. VAN DER Mee, Explicit solutions of the cubic matrix nonlinear Schrödinger equation, Inverse Problems 24:02520 (16 pp.) (2008).

36. A. Fratalocchi, C. Conti, G. Ruocco, and S. Trillo, Free-energy transition in a gas of noninteracting nonlinear wave particles, Phys. Rev. Lett. 101(4):044101 (4 pp.) (2008).

37. B. Prinari, M. J. Ablowitz, and G. Biondini, Inverse scattering transform for the vector nonlinear Schrödinger equation with nonvanishing boundary conditions, J. Math. Phys. 47:063508 (33 pp.) (2006).

38. B. Prinari, G. Biondini, and A.D. Trubatch, Inverse scattering transform for the multicomponent nonlinear Schrödinger equation with nonzero boundary conditions at infinity, Stud. Appl. Math. 126:245-302 (2011).

39. F. DEMONTIS and C. VAN DER MEe, Wave operators for defocusing matrix Zakharov-Shabat systems with potentials not vanishing at infinity, Serdica Math. J. 36:265-284 (2010).

40. H. Dym, Linear Algebra in Action, Graduate Studies in Mathematics, Vol. 78, American Mathematical Society, Providence, RI, 2007. 
41. A. Dimakis and F. Müller-Hoissen, Solutions of matrix NLS systems and their discretizations: a unified treatment, Inverse Problems 26:095007 (55 pp.) (2010).

42. C. Schiebold, Solutions of the sine-Gordon equation coming in clusters, Revista Matemática Complutense 15:265-325 (2002).

43. C. Schiebold, Cauchy-type determinants and integrable systems, Linear Algebra Appl. 433:447-475 (2010).

44. M. Klaus, On the Zakharov-Shabat eigenvalue problem, Mathematical Studies in Nonlinear Wave Propagation, Contemporary Mathematics, Vol. 379, pp. 21-45, American Mathematical Society, Providence, RI (2005).

45. G. PólYA and G. SzEGÖ, Aufgaben und Lehrsätze aus der Analysis, II, Springer, Berlin and Heidelberg, 1971; also: G. Pólya and G. Szegö, Problems and Theorems in Analysis, II. Theory of Functions, Zeros, Polynomials, Determinants, Number Theory, Geometry, Classics in Mathematics, Springer, Berlin, 1998.

UNIVERSITY OF CAGLIARI

University of Colorado at Colorado Springs,

University of SAlento \& SEZione InfN Lecce

UNIVERSITY OF CAGLIARI

University of Salento \& Sezione InfN Lecce

(Received August 2, 2012) 